\title{
VALUATIONS, PRIMES AND IRREDUCIBILITY IN POLYNOMIAL RINGS AND RATIONAL FUNCTION FIELDS
}

BY

\author{
RON BROWN (1)
}

ABSTRACT. The set of extensions of the valuation $v$ on a linearly compact (i.e. maximal) field $F$ to the polynomial ring $F[x]$ is shown to depend only on the value group and residue class field of $v$. By a method related to Mac Lane's construction of (rank one) valuations on polynomial rings, a determining invariant is associated with each such extension, called its "signature". Very roughly, a signature is a pair of sequences, one in the algebraic closure of the residue class field of $v$ and one in the divisible closure of the value group of $v$. Signatures are also associated with various mathematical objects by means of the extensions of the above sort which naturally arise from them. For example, the set of nonconstant monic irreducible polynomials in $F[x]$, the set of all finite Harrison primes of the polynomial ring of a global field, and the set of equivalence classes of valuations on the field of rational functions over a global field are each shown to be bijective with a simple set of signatures. Moreover, these objects are studied by means of their associated signatures. For example, necessary and sufficient conditions for irreducibility in $F[x]$ are given, independent of the language of signatures.

1. Introduction. Let $v$ be a discrete rank one valuation on a field $F$. We study the extensions of $v$ to a valuation on the polynomial ring $F[x]$, assigning to each extension a determining invariant. (For valuations on commutative rings see, for example, Bourbaki [2, p. 101].) Our approach has considerable overlap with Mac Lane's construction of the (rank one) valuations on $F[x]$ extending $v$ [15]. In particular, the underlying method consists of approximating a valuation on $F[x]$ by means of the valuations on an associated sequence of simple algebraic extensions of $F$.

We call the determining invariant associated with an extension of $v$ to $F[x]$ its "signature". Abstractly, signatures are defined entirely in terms of the residue

Presented to the Society, August 28, 1969 under the title Valuations, primes and irreducibility in rings of polynomials and rational functions; received by the editors November 19, 1970.

AMS (MOS) subject classifications (1970). Primary 12J10, 12E05, 12B05, 13A15; Secondary 12A20, 12F05, 12F 20, 12B 10, 12J 20, $13 \mathrm{~F} 20$.

Key words and phrases. Valuation, Harrison prime, discrete rank one valuation, polynomial ring, rational function field, irreducibility, Legendre polynomial, Eisenstein criterion, key polynomial, complete field, Henselian field, linearly compact field, maximal field, global field, algebraic extension, ramification, inductive value, limit value.

(1) Work on this paper was done over an extended period during which the author was variously an NSF fellow and an NAS-ONR research associate at the University of Oregon, and a postdoctoral fellow (partially supported by the Canadian NRC) at Simon Fraser University. 
class field and value group of $v$; roughly, a signature consists of two sequences, one in the algebraic closure of the residue class field of $v$ and one in the divisible closure of the value group of $v$. An axiomatic approach is used for assigning signatures to extensions; this allows a rapid exposition of our main results. In order that the axioms not appear too unmotivated we present in $\$ 2$ a leisurely sketch of the construction of the signatures associated with an important set of valuations on $Q[x]$ ( $Q$ = rational numbers), namely those associated with finite Harrison primes [10, p. 18]. The definitions and axioms for "associated signatures" appear in $\$ 3$, along with the statement of a lemma fundamental to our approach and having no analogue in [15]. Given an irreducible polynomial $f$ and an extension $w$ of $v$ to $F[x]$, it describes when the value of $w$ at $f$ determines the value of $w$ at every polynomial of degree less than that of $f$.

In $\$ 4$ we prove that when $F$ is topologically complete in the valuation topology of $v$ (abbreviated, complete), then the set of extensions of $v$ to $F[x]$ is bijective with an easily described set of signatures depending only on the residue class field and value group of $v$. We further describe how to recover an extension from its associated signature.

Our first applications are based on the observation that for any prime ideal $p$ of $F[x]$, the extensions $w$ of $v$ to $F[x]$ with $p=w^{-1}(\infty)$ are naturally bijective with the extensions of $v$ to the field of fractions of $F[x] / p$. From the case $p=\{0\}$, we obtain a characterization in terms of signatures of the extensions of $v$ to the field of rational functions $F(x)$. From the case $p \neq\{0\}$ (so that $F[x] / p$ is an algebraic extension of $F$ ), we develop several of the basic known facts about algebraic extensions of complete fields as corollaries to our description of valuations on $F[x]$.

Similar reasoning gives, again for $F$ complete, a bijection from the set of nonconstant monic irreducible polynomials in $F[x]$ to a certain easily described set of signatures. This bijection is applied to give necessary and sufficient conditions for irreducibility in $F[x]$ which are independent of the language of associated signatures. While closely related to Mac Lane's generalizations of "polygonal irreducibility criteria"' (e.g. Eisenstein's irreducibility criterion) $[17, \S 3]$, our conditions do not apparently follow from them without the fundamental lemma. Our remarks about irreducibility and algebraic extensions appear in $\$ 5$; we also make some remarks there about the not necessarily complete case.

In $\$ 6$ we compute the extensions of $v$ to $F[x]$ no longer assuming that $F$ is complete. In applications, the valuations and finite Harris on primes of $F[x]$ and $F(x)$ are computed in terms of signatures for $F$ a global field. We also compute the finite Harrison primes of $R[x]$ where $R$ is a subring of a global field (e.g. $R=Z$, the integers). 
All the results of $\$ \$ 3,4$ and 5 about fields complete at a discrete rank one valuation generalize fully to arbitrary linearly compact (alias, maximal) fields. This generalization is sketched in $\$ 7$. Incidentally, it would have been possible to proceed initially in great generality, starting only with an arbitrary valuation on a commutative ring (see [3, p. 78] for an example of grotesque generality). Most of the results developed, however, would be more easily obtained as corollaries to the theory developed here, just as the noncomplete case in $\$ 6$ is developed as a corollary of the complete case in $\$ 4$. An example of such an application occurs in $\$ 9$, where we take the briefest look at polynomials in several variables.

In $\$ 8$ we give a proof of the fundamental lemma for linearly compact fields.

We have delayed until our tenth and last section giving detailed acknowledgements of, and connections with, Mac Lane's work ([15], [16], [17]). This was mainly for expository reasons: the connections are somewhat technical and emerge most clearly from a developed theory. This is the only section for which [15] is a prerequisite.

We now list for reference some notations that will be used throughout; most will be reintroduced before being used again.

"deg $f$ " denotes the degree of a polynomial $f . F^{\times}$denotes the multiplicative group of nonzero elements of a field $F$.

Let $n$ be a nonnegative integer or $\infty$. The symbol $\left\langle a_{i}\right\rangle_{0 \leq i<n}$ (or, more compactly, $\left.\left\langle a_{i}\right\rangle_{i<n}\right)$ denotes the sequence defined on the set of nonnegative integers less than $n$ whose value at $i$ is $a_{i}$; we call $n$ the lengt $b$ of the sequence. We allow the sequence of length zero, i.e. the "empty sequence". If $n<\infty,\left\langle a_{i}\right\rangle_{i \leq n}$ has the obvious meaning.

We use additive notation for valuations. For any ring valuation $v: R \rightarrow$ $\Gamma \cup\{\infty\}[2$, p. 101] we call

$$
\begin{array}{ll}
A_{v}=\{a \in R \mid v(a) \geq 0\} & \text { the valuation ring of } v, \\
P_{v}=\{a \in R \mid v(a)>0\} & \text { the prime ideal of } v, \\
\Gamma_{v}=\Gamma \cap v(R) & \text { the value monoid of } v, \\
k_{v}=A_{v} / P_{v} & \text { the residue class ring of } v, \\
v^{-1}(\infty) . & \text { the infinity ideal of } v .
\end{array}
$$

The valuation $v$ is equivalent to a valuation $v^{\prime}$ on $R$ if and only if there is an order isomorphism from $\Gamma_{v} \cup\{\infty\}$ to $\Gamma_{\nu}{ }^{\prime} \cup\{\infty\}$ whose composition with $v$ is $v^{\prime}$.

Note that $\infty$ is used both as an index for sequences and as an infinity for valuations, depending on context. 
It is a great pleasure to thank D. K. Harrison and Hoyt Warner for reading various versions of this paper and pointing out many slips and obscurities. I am further in debt to D. K. Harrison for encouragement and generously given advice. This paper has considerable overlap with the author's doctoral dissertation [3], to which we occasionally refer for material peripheral to the main argument.

2. Prototype example: $Q[x]$. This paper originated in the study of this example, so it is germinal as well as prototypal. $Q$ denotes the rational numbers, either as a field or as an additive ordered group, depending on context.

We will compute the valuations on $Q[x]$ whose value monoids are groups and whose residue class rings are locally finite fields (i.e. are fields which are the union of their finite subfields). We call such valuations prime valuations since the mapping $w \mapsto \mathfrak{P}_{w}=\{a \in Q[x] \mid w(a)>0\}$ induces a bijection from the set of equivalence classes of prime valuations on $Q[x]$ to the set finite Harrison primes of $Q[x]$ (cf. [10, p. 18]).

So let $w$ be a prime valuation on $Q[x]$. Let $v$ denote the restriction of $w$ to $Q$; if $p$ denotes the characteristic of the residue class field $k_{w}$, then $v$ is the $p$-adic valuation on $Q$.

We wish initially to construct a sequence $S(w)=\left\langle\theta_{i}, q_{i}\right\rangle_{i<n}$ of ordered pairs in $k_{w}^{\times} \times \Gamma_{w}$. (Strictly speaking we should write $\left\langle\left(\theta_{i}, q_{i}\right)\right\rangle_{i<n}$ but our abbreviation should cause no confusion.) One's first thought might be to set $q_{i}=w\left(x^{i}\right)$ and $\theta_{i}=x^{i}+p_{w}$ for each $i<\infty$. However, the resulting sequence, even when it made sense, would not in general tell one much about $w$. Rather, using a construction due to Mac Lane [15], we replace the sequence $\left\langle x^{i}\right\rangle_{i<\infty}$ implicitly used above by a sequence $g(w)$ of polynomials which reflects the additional structure that $w$ puts on $Q[x]$ (much as the Gram-Schmidt orthogonalization process replaces a linear basis on an inner product space by one which reflects the inner product). We then use $g(w)$ to define $S(w)$.

Let $g_{0}=x, g_{1}, \cdots, g_{m}$ be a finite sequence of nonconstant polynomials with $\operatorname{deg} g_{s}$ dividing $\operatorname{deg} g_{s+1}$ for all $s<m$. Each $f \in Q[x]$ has a unique expansion

$$
\sum a_{i_{0}} \cdots i_{m} g_{0}^{i_{0}} \cdots g_{m}^{i_{m}}
$$

where the coefficients $a_{i_{0}} \cdots i_{m}$ are in $Q$ and

$$
0 \leq i_{s}<\left(\operatorname{deg} g_{s+1}\right) /\left(\operatorname{deg} g_{s}\right) \quad(0 \leq s<m) .
$$

(This is because for each integer $n \geq 0$ there are unique $i_{s}$ as in (2) with $n=\Sigma i_{s} \operatorname{deg} g_{s}$, i.e. with

$$
g_{0}^{i_{0}} \cdots g_{m}^{i_{m}}
$$

of degree exactly $n$.) We say that the polynomial (1) is $w$-bomogeneous in 
$\left\langle g_{i}\right\rangle_{i \leq m}$ if and only if every nonzero term in the expansion (1) has the same value, and each coefficient $a_{i_{0}} \ldots i_{m}$ is the product of an integral power of $p$ with an element of $\{0,1, \cdots, p-1\}$. (The value of an element of a valued ring is its image under the valuation.)

We now define $g(w)=\left\langle g_{i}\right\rangle_{i<n}$ to be the unique sequence of polynomials of maximal length $n$ (where $0 \leq n \leq \infty$ ) such that each polynomial has finite value, $g_{0}=x$ if $0<n$, and for any positive integer $m+1<n$,

(I) $\operatorname{deg} g_{m+1}$ is divisible by $\operatorname{deg} g_{m}$;

(II) $g_{m+1}$ is monic and $w$-homogeneous in $\left\langle g_{i}\right\rangle_{i \leq m}$;

(III) $w\left(g_{m+1}\right)$ is greater than the value of any of the nonzero terms of $g_{m+1}$, when $g_{m+1}$ is expanded as in (1);

(IV) $g_{m+1}$ is a polynomial of minimal degree satisfying (III).

The uniqueness of the sequence $g(w)$ is easily verified. (If $0<m+1<n$, then the difference of any two distinct candidates for $g_{m+1}$ would be a polynomial of degree less than $\operatorname{deg} g_{m+1}$ satisfying (III); but this contradicts (IV).) Notice that by (IV), the value of any polynomial of degree less than $\operatorname{deg} g_{m+1}$ is equal to the minimum of the values of its terms, when expanded as in (1). Thus, while $g(w)$ is not a basis for $F[x]$ as an $F$-module, for each $0<m<n$ the set of products (3) approximates an "orthobas is" in the sense of MacKenzie-Whaples [14].

Note that we allow $n=0$ above; this case arises when $w(x)=\infty$.

Before constructing $S(w)$, it is helpful to notice

Lemma. There is a unique order isomorphism of $\Gamma_{w}$ into $Q$ which maps $w(p)$ to 1 . Here, $Q$ denotes the additive ordered group of rational numbers.

Proof (sketch). We first identify the cyclic subgroup of $\Gamma_{w}$ generated by $w(p)$ with the integers, $Z$. It then suffices to show that each element of $\Gamma_{w}$ is "commensurable with $Z$ ", i.e. has a nonzero integral multiple in $Z$. Just suppose that there is a polynomial $f$ of least degree whose value is finite and not commensurable with $Z$. One then can verify that $w(f)$ cannot have an inverse in $\Gamma_{w}$, contradicting that $\Gamma_{w}$ is a group. (Use here the fact that by the choice of $f$ the value of a polynomial

$$
\sum r_{i} f^{i} \quad\left(\operatorname{deg} r_{i}<\operatorname{deg} f\right)
$$

must equal the minimum of the values of its terms.)

We now construct $S(w)$. For each $i<n$, set $q_{i}=w\left(g_{i}\right)$. If $w\left(g_{i}\right)=w\left(p^{s}\right)$, it is natural to set $\theta_{i}=p^{-s} g_{i}+p_{w}$. To proceed in general, we first set (recall $v=w \mid Q$ and $Z=$ integers)

$$
\Gamma_{m}=\Gamma_{\nu}+\sum_{i<m} Z \cdot q_{i} \quad(0 \leq m \leq n) .
$$

Notice that $w$ gives a bijection between $\Gamma_{n}$ and the set of all finite products 


$$
p^{r_{n}} \prod_{i<n} g_{i}^{r_{i}}
$$

where only finitely many of the integers $r_{i}$ are nonzero and

$$
0 \leq r_{i}<\left(\Gamma_{i+1}: \Gamma_{i}\right) \quad(i<n) .
$$

Here $\left(\Gamma_{i+1}: \Gamma_{i}\right)$ denotes the index of $\Gamma_{i}$ in $\Gamma_{i+1}$. We then set $\theta_{m}=t_{m} g_{m}+p_{w}$ for each $m<n$, where $t_{m}$ is the unique product (5) with $w\left(t_{m}\right)=-w\left(g_{m}\right)$. We call $S(w)=\left\langle\theta_{i}, q_{i}\right\rangle_{i<n}$ the "presignature" of $w$.

A technical point. By strict analogy with the definition of the $\Gamma_{m}$, we define

$$
k_{m}=k_{\nu}\left[\left\{\theta_{i} \mid i<m\right\}\right] \quad(0 \leq m \leq n) .
$$

Note that the degree $\left[k_{m+1}: k_{m}\right]$ of each extension $k_{m+1} / k_{m}$ is finite since $k_{w}$ is a locally finite field. The fact that $\left(\Gamma_{m+1}: \Gamma_{m}\right) w\left(g_{m}\right) \in \Gamma_{m}$ and that $\theta_{m}=$ $t_{m} g_{m}+p_{w}$ satisfies a polynomial of degree $\left[k_{m+1}: k_{m}\right]$ over $k_{m}$ can be shown (nontrivially!) to imply that for $0<m+1<n$

$$
\operatorname{deg} g_{m+1}=\left[k_{m+1}: k_{m}\right]\left(\Gamma_{m+1}: \Gamma_{m}\right) \operatorname{deg} g_{m}
$$

and hence by induction

$$
\operatorname{deg} g_{m}=\left[k_{m}: k_{v}\right]\left(\Gamma_{m}: \Gamma_{v}\right) \quad(m<n) .
$$

Notice that the sequence $\left\langle\theta_{i}, q_{i}\right\rangle_{i<n}$ can be defined in the above manner assuming only that each polynomial in the sequence $\left\langle g_{i<n}\right\rangle_{i<n}$ has finite value. With this understanding, (I) and (IV) in the definition of $g(w)$ above can be replaced by (I'). Condition (III) then becomes

$$
q_{m+1}>\left[k_{m+1}: k_{: n}\right]\left(\Gamma_{m+1}: \Gamma_{m}\right) q_{m}
$$

or equivalently

$$
\left\langle q_{i} /\left[k_{i}: k_{v}\right]\left(\Gamma_{i}: \Gamma_{\nu}\right)\right\rangle_{i<n} \text { is strictly increasing. }
$$

This is because the right-hand side of (7) is precisely the value of $g_{m}\left[k_{m+1}: k_{m}\right]\left(\Gamma_{m+1}: \Gamma_{m}\right)$ which by (6) is one of the nonzero terms of $g_{m+1}$ when expanded as in (1).

One more step. We free $S(w)$ from its dependence on the notation of $w$ (recall $S(w)$ is a sequence of pairs in $\left.k_{w}^{\times} \times \Gamma_{w}\right)$. The values $q_{i}$ are no problem since by the lemma above they can be uniquely identified $w$ ith rational numbers. In order to regard the $\theta_{i}$ as lying is some fixed algebraic closure of $Z_{p}$ (= the field with $p$ elements), call it $Z_{p}^{\mathrm{alg}}$, we must choose an isomorphism of $k_{w}$ into $Z_{p}^{\mathrm{alg}}$. Since any two such isomorphisms differ by an automorphism of $Z_{p}^{\text {alg }}, S(w)$ determines a unique " $p$-signature" in the sense of the following definition.

Definition. A p-signature is an equivalence class ("equivalence" defined below) of sequences $\left\langle\theta_{i}, q_{i}\right\rangle_{i<n}$ of ordered pairs in $\left(Z_{p}^{\mathrm{alg}}\right)^{\times} \times Q$ which satisfy 
(III') above (with the obvious notation). Two such sequences $\left\langle\theta_{i}, q_{i}\right\rangle_{i<n}$ and $\left\langle\theta_{i}^{\prime}, q_{i}^{\prime}\right\rangle_{i<n}$ are equivalent if and only if $n=n^{\prime}, q_{i}=q_{i}^{\prime}$ for all $i<n$, and there is an automorphism of $Z_{p}^{\text {alg }}$ carrying each $\theta_{i}$ to $\theta_{i}^{\prime}$.

We avoid developing the ideas of this section further until we can proceed in greater generality, except to state

Theorem. Assigning to each prime valuation of $Q[x]$ its corresponding $p$-signature induces a bijection from the set of equivalence classes of prime valuations on $Q[x]$ to the set of $p$-signatures, where $p$ ranges over all prime numbers.

The proof of this theorem will give both an internal picture of the valuation associated with a given $p$-signature and an external picture of the space of all such valuations (e.g. see (6.8) below).

We close this section with a concrete example. Let $v: Q \rightarrow Z \cup\{\infty\}$ be the $p$-adic valuation and let

$$
w: f \mapsto v(f(\alpha)) \quad(f \in Q[x])
$$

be the valuation induced by $v$ and an element $\alpha$ of $Q$, say with $p$-adic expansion $\sum a_{i} p^{i}\left(0 \leq a_{i}<p\right.$ for all $\left.i\right)$. Let $n$ be the number of nonzero terms of $\sum a_{i} p^{i}$, and for each $i<n$, let $a_{\sigma(i)} p^{\sigma(i)}$ be the $(i+1)$ th such term. Then

$$
g(w)=\left\langle x+\sum_{i<m} a_{\sigma(i)} p^{\sigma(i)}\right\rangle_{m<n} \text { and } S(w)=\left\langle a_{\sigma(i)}+p Z, \sigma(i)\right\rangle_{i<n} .
$$

3. Signatures and the Fundamental Lemma. We now generalize the notion of a $p$-signature introduced in $\$ 2$. In this generalization we allow "sequences of length $\infty+1$ ", i.e. sequences of the form $\left\langle a_{i}\right\rangle_{i \leq \infty}$ or, equivalently, $\left\langle a_{i}\right\rangle_{i<\infty+1}$. We are regarding $\infty$ and $\infty+1$ as formal symbols with $n<\infty<\infty+1$ for all integers $n$. (In $\$ 7$ we will regard sequences as defined on initial segments of the ordinal numbers. In that context sequences of length $\infty+1$ appear more naturally as sequences defined on the set of ordinal numbers less than the successor of the first infinite ordinal number.)

Let $k$ be a field and $\Gamma$ be a linearly ordered abelian group (abbreviated, ordered group). A presignature over $(k, \Gamma)$ is a sequence of ordered pairs $S=$ $\left\langle\theta_{i}, q_{i}\right\rangle_{i<n}$ where $0 \leq n \leq \infty+1$ and where the $\theta_{i}$ all lie in some field containing $k$ and the $q_{i}$ all lie in some ordered group containing $\Gamma$. Given such a presignature $S$, we define nondecreasing sequences of integral domains and ordered semigroups

$$
k=k_{0} \subseteq k_{1} \subseteq \cdots \subseteq k_{n}=k_{S}, \quad \Gamma=\Gamma_{0} \subseteq \Gamma_{1} \subseteq \cdots \subseteq \Gamma_{n}=\Gamma_{S},
$$

where, for each $m \leq n, k_{m}$ is the ring extension of $k$ generated by $\left\{\theta_{i} \mid i<m\right\}$ and $\Gamma_{m}$ is the semigroup extension of $\Gamma$ generated by $\left\{q_{i} \mid i<m\right\}$. If $S^{\prime}=$ 
$\left\langle\theta_{i}^{\prime}, q_{i}^{\prime}\right\rangle_{i<n}$ is a second presignature over $(k, \Gamma)$, we say it is equivalent to $S$ if $n=n^{\prime}$ and, with the obvious notation,

(i) there is an order isomorphism of $\Gamma_{S}$ onto $\Gamma_{S}$ ' leaving $\Gamma$ elementwise fixed and taking each $q_{i}$ to $q_{i}^{\prime}$;

(ii) there is an isomorphism of $k_{S}$ onto $k_{S}$ ' leaving $k$ elementwise fixed and taking each $\theta_{i}$ to $\theta_{i}^{\prime}$.

Notice that the isomorphisms of (i) and (ii) above are uniquely determined. An equivalence class of presignatures will usually be denoted by one of its members.

(3.1) Definition. A signature over $(k, \Gamma)$ is an equivalence class of presignatures $S$ over $(k, \Gamma)$ with (notation for $S$ as above)

(i) if $i<n$, then $\left[k_{i}: k\right]\left(\Gamma_{i}: \Gamma\right)<\infty$, and the sequence

$$
\left\langle q_{i} /\left[k_{i}: k\right]\left(\Gamma_{i}: \Gamma\right)\right\rangle_{i<n}
$$

is strictly increasing;

(ii) if $i<n$, then $\theta_{i}$ is nonzero if and only if $q_{i}$ is "commensurable with $\Gamma$ "' (i.e. for some positive integer $n, n q_{i} \in \Gamma$ ).

Recall that $\left[k_{i}: k\right]$ denotes the dimension of $k_{i}$ over $k$ and $\left(\Gamma_{i}: \Gamma\right)$ denotes the index of $\Gamma$ in $\Gamma_{i}$. The elements of the sequence (9) are regarded as lying in the "divisible closure" of $\Gamma_{S}$, in the following sense.

(3.2) Lemma (and definition). Let $\Lambda$ be a submonoid of an ordered group. There is a divisible ordered group containing $\Lambda$, which we denote by $\Lambda^{\mathrm{div}}$ and call the divisible closure of $\Lambda$, sucb that every element of $\Lambda^{\mathrm{div}}$ is commensurable with $\Lambda$, i.e. has an integral multiple which is equal to the difference of two elements of $\Lambda . \Lambda^{\mathrm{div}}$ is unique up to a (uniquely determined) isomorphism leaving $\Lambda$ elementwise fixed.

Proof [21, p. 8]. Actually, we may set $\Lambda^{\mathrm{div}}=\Lambda \otimes_{Z} Q$.

Let $S=\left\langle\theta_{i}, q_{i}\right\rangle_{i<n}$ be a signature over $(k, \Gamma)$. Notice that each $\theta_{i}$ is nonzero and algebraic over $k$, except possibly the last one (if there is a last one). Similarly, each $q_{i}$ is commensurable with $\Gamma$ except possibly a last one. Further, $\theta_{i}$ is zero if and only if the corresponding $q_{i}$ is not commensurable with $\Gamma$. If $n=\infty+1$, then $q_{\infty}$ is larger than every element of $\Gamma$ and hence is not commensurable with $\Gamma$. For any $m$, if $0<m+1<n$, then

$$
q_{m+1}>\left[k_{m+1}: k_{m}\right]\left(\Gamma_{m+1}: \Gamma_{m}\right) q_{m} .
$$

The reader can now check that the conditions on $S$ listed in this paragraph are sufficient to guarantee that the equivalence class of a presignature satisfying them be a signature. In particular, the $p$-signatures of $\$ 2$ may be identified with certain of the signatures over $\left(Z_{p}, Z\right)$. 
Incidentally, it is easy to verify that the collection of signatures over $(k, \Gamma)$ form a set (use the paragraph above). Indeed one can construct a field $k^{\prime} \supset k$ and an ordered group $\Gamma^{\prime} \supset \Gamma$ such that every presignature satisfying (i) of (3.1) is equivalent to a presignature with values in $k^{\prime} \times \Gamma^{\prime}$.

We introduce a useful variant of Definition (3.1).

(3.3) Definition. Let $S=\left\langle\theta_{i}, q_{i}\right\rangle_{i<n}$ be a presignature over $(k, \Gamma)$. We call $\left[k_{S}: k\right]\left(\Gamma_{S}: \Gamma\right)$ the degree of $S$, denoted "deg $S$ ". If $\operatorname{deg} S=\infty$, we set aug $S$ $=S$. If $\operatorname{deg} S<\infty$, we set aug $S=\left\langle\theta_{i}, q_{i}\right\rangle_{i \leq n}$ where $\theta_{n}=0$ and $q_{n}=\infty$. If $S^{\prime}$ is a second presignature over $(k, \Gamma)$, we say aug $S$ is equivalent to aug $S^{\prime}$ if $S$ is equivalent to $S^{\prime}$. If the equivalence class of $S$ is a signature, we call the equivalence class of aug $S$ an augmented signature over $(k, \Gamma)$. Finally, we write $k_{\text {aug }} S=k_{S}, \Gamma_{\text {aug }} S=\Gamma_{S}$, and $\operatorname{deg}(\operatorname{aug} S)=\operatorname{deg} S$.

As with signatures, augmented signatures will usually be denoted by a representative "augmented presignature". Notice that any augmented signature has the form $\left\langle\theta_{i}, q_{i}\right\rangle_{i<n}$ where $n \leq \infty+1$ (for if the signature $S$ has length $\infty+1$, it must have infinite degree-see the paragraph following (3.2)). We may define the sequences of $\Gamma_{m}$ and $k_{m}$ (see (8)) for augmented signatures over $(k, \Gamma)$ at least when $m<n$. (Thus $\Gamma_{n}$ is not defined when $q_{n-1}=\infty$.)

Note that the map $S \mapsto$ aug $S$ gives a bijection from signatures to augmented signatures.

Now let $v$ be a discrete rank one valuation on a field $F$. We shall define when a signature over $\left(k_{v}, \Gamma_{v}\right)$ is "associated" with an extension of $v$ to $F[x]$. This definition will depend on the choice, which we now make once and for all, of a system $A$ of representatives in $F^{\times}$for $\Gamma_{v}$ (so $v$ maps $A$ bijectively onto $\left.\Gamma_{v}\right)$ and a system $B$ of coset representatives in $F^{\times}$for $F^{\times} /\left(1+p_{v}\right)$. We further assume for convenience that $1 \in A \cap B$.

In $\S 2$ where $v$ was the $p$-adic valuation on $Q$, we implicitly took $A$ to be the set of integral powers of $p$ and $B$ to be $\left\{s p^{t} \mid s\right.$ and $t$ integers with $0<s$ $<p\}$.

The important group $F^{x} /\left(1+p_{v}\right)$ will be discussed further below.

Recall a concept introduced in $\$ 2$. Suppose $w$ is an extension of $v$ to $F[x]$. Let $g_{0}=x, g_{1}, \cdots, g_{m}$ be a finite sequence in $F[x] \backslash F$ with deg $g_{s}$ dividing $\operatorname{deg} g_{s+1}$ for all $s<m$. Each element of $F[x]$ has a unique expansion

$$
\sum b_{i} g_{0}^{i_{0}} \cdots g_{m}^{i_{m}} \quad\left(i=\left(i_{0}, \cdots, i_{m}\right)\right)
$$

where the coefficients $b_{i}$ are in $F$ and $0 \leq i_{s}<\left(\operatorname{deg} g_{s+1}\right) /\left(\operatorname{deg} g_{s}\right)(0 \leq s,<m)$. Following Mac Lane [15], we say a polynomial is $w$-homogeneous in $\left\langle g_{i}\right\rangle_{i \leq m}$ if when expanded as in (10), each coefficient $b_{i}$ is either zero or in $B$ and each nonzero term of (10) has the same value. (The value of an element of a valued ring is its image under the valuation.) 
We use the notation for signatures above, setting $(k, \Gamma)=\left(k_{v}, \Gamma v\right)$.

(3.4) Definition. Let $w$ be an extension of $v$ to a valuation on $F[x]$. We say a (possibly augmented) signature $S=\left\langle\theta_{i}, q_{i}\right\rangle_{i<n}$ is associated with $w$ if and only if there is a sequence $\left\langle g_{i}\right\rangle_{i<n}$ of monic polynomials in $F[x]$ (which we call a generating sequence for $w$ and $S$ ) with

(i) if $0<n$, then $g_{0}=x$;

(ii) if $m<n, \operatorname{deg} g_{m}=\left[k_{m}: k_{\nu}\right]\left(\Gamma_{m}: \Gamma_{\nu}\right)$;

(iii) if $0<m+1<n$, then $g_{m+1}$ is $w$-homogeneous in $\left\langle g_{i}\right\rangle_{i \leq m}$;

(iv) if $m<n$, then $q_{m}=w\left(g_{m}\right)$;

(v) if $m<n$ and $q_{m}$ is finite and commensurable with $\Gamma_{v}$, then we can write

$$
\theta_{m}=\left(a \prod_{i \leq m} g_{i}^{r_{i}}\right) g_{m}+\wp_{w}
$$

where $a \in A$ and $0 \leq r_{i}<\left(\Gamma_{i+1}: \Gamma_{i}\right)$ for $i \leq m$.

Also, we say a sequence of monic polynomials $\left\langle g_{i}\right\rangle_{i<n}$ in $F[x]$ is a generating sequence for $w$ if and only if there exists a (possibly augmented) signature $S$ such that $\left\langle g_{i}\right\rangle_{i<n}$ is a generating sequence for $w$ and $S$.

The above definition says that if $S$ is associated with $w$, then a represenative presignature for $S$ can be found with the $q_{i}$ in $\Gamma_{w}$ and the $\theta_{i}$ in $k_{w}$.

Notice that for each $m<n$ with $q_{m}$ finite and commensurable with $\Gamma_{v}$, the corresponding choice of $a$ and $r_{i}(i \leq m)$ in (v) of (3.4) above is uniquely determined by $v(a)+\Sigma r_{i} q_{i}=-q_{m}$ (this must be true since $\theta_{m} \neq 0$ ). Further, if $q_{m}$ is not finite and commensurable with $\Gamma_{v}$, then $\theta_{m}=0$ (cf. (3.1)). Hence (iv) and (v) of (3.4) guarantee that $w$ and $\left\langle g_{i}\right\rangle_{i<n}$ completely determine $S$. Something much stronger is true. The next lemma says that $S$ determines $\left\langle g_{i}\right\rangle_{i<n}$ independently of $w$, and that for each $m<n$, the fact that $\left\langle\theta_{i}, q_{i}\right\rangle_{i<m}$ is associated with $w$ can be deduced from the fact that $w$ assigns to $g_{m}$ a sufficiently large value.

(3.5) Fundamental Lemma. Suppose $F$ is complete. Let $S=\left\langle\theta_{i}, q_{i}\right\rangle_{i<n}$ be any signature over $\left(k_{v}, \Gamma_{v}\right)$ of finite degree (cf. (3.1) and (3.3)). Then there exists a unique monic nonconstant polynomial $f \in F[x]$ sucb that, for any extension $w$ of $v$ to $F[x]$, the following are equivalent (with the usual notation):

(i) $S$ is associated with $w$;

(ii) $w$ bas a unique generating sequence of length $n+1$ and $f$ is its nth term;

(iii) $w(f)>\left[k_{S}: k_{i}\right]\left(\Gamma_{S}: \Gamma_{i}\right) q_{i}$ for all $i<n$.

We call $f$ the generator of $S$.

(3.6) Examples. (A) $x$ is the generator of the sequence of length zero.

(B) Suppose $f=\Sigma b_{i} x^{i}$ is a monic nonconstant polynomial all of whose nonzero coefficients are in $\{b \in B \mid v(b)=0\}$. If $\Sigma\left(b_{i}+p_{v}\right) x^{i}$ is an irreducible 
polynomial over $k_{v}$ with a nonzero root $a$ in some extension of $k_{v}$, then $f$ is the generator of the signature of length one whose only term is $(a, 0)$.

(C) Suppose $v(b)$ is the least positive element of $\Gamma_{v}, b \in B$, and $b^{-1} \in A$. Then for any positive integer $n, x^{n}+b$ is the generator of the signature of length one whose only term is $(-1, v(b) / n)$.

The proof of (3.5) is a complicated but basically straightforward counting argument. In order to proceed directly to the main results of this paper, we refer the reader to $\$ 8$ for its proof.

A final remark. All of the above definitions make sense when $v$ is the trivial valuation on $F$. Since $F$ is linearly compact for the trivial valuation, all of our results hold in this case as well as the discrete rank one case (cf. $\$ 7$ ). These results are in every case familiar, e.g. Lemma (3.5) above becomes the usual bijection between the monic nonconstant irreducible polynomials over $F$ and the $F$-conjugacy classes of elements in the algebraic closure of $F$. (For another example, compare Corollary (4.3) below with [8, p. 46].)

4. Valuations on the polynomial ring and rational function field of a complete field. Throughout this section $F$ will denote a field complete at a discrete rank one valuation $v . A$ and $B$ again denote systems of representatives for $\Gamma_{v}$ and $F^{X} /\left(1+p_{v}\right)$, respectively, with $1 \in A \cap B$. Associated signatures and generating sequences are implicitly assumed to be defined with respect to the choice of $A$ and $B$.

(4.1) Main Theorem. Each extension of $v$ to $F[x]$ bas associated with it a unique augmented signature over $\left(k_{v}, \Gamma_{v}\right)$. This association induces a bijection from the set of equivalence classes of extensions of $v$ to $F[x]$ onto the set of augmented signatures over $\left(k_{v}, \Gamma_{v}\right)$.

Recall that two valuations on a ring are equivalent if they differ by an isomorphism of their value monoids (cf. $\$ 1$ ).

This section is devoted to the proof of (4.1) and its "supplement" (4.2), which describes how to recover a valuation from its augmented signature. As a corollary, we also compute in terms of signatures the set of extensions of $v$ to the field of rational functions $F(x)$.

(4.2) Supplement to the Main Theorem. Let $S=\left\langle\theta_{i}, q_{i}\right\rangle_{i<n}$ be an augmented signature over $\left(k_{v}, \Gamma_{v}\right)$. For each $m<n$, let $g_{m}$ denote the generator of the signature $\left\langle\theta_{i}, q_{i}\right\rangle_{i<m}(c f .(3.5))$.

(A) Let $D_{S}$ denote the set of all products

$$
\prod_{i<n} g_{i}^{r_{i}}
$$


where only a finite number of the $r_{i}$ are nonzero and

$$
0 \leq r_{i}<\left[k_{i+1}: k_{i}\right]\left(\Gamma_{i+1}: \Gamma_{i}\right) \quad(0<i+1<n) .
$$

Then $D_{S}$ is a basis for $F[x]$ as an F-module. Indeed, for any nonnegative number $n$ there are unique $r_{i}$ as in (12) such that the product (11) bas degree exactly $n$ (since the right-hand side of (12) equals $\left(\operatorname{deg} g_{i+1}\right) /\left(\operatorname{deg} g_{i}\right)$ ).

(B) For any product (11) above, let us set

$$
v_{s}\left(\prod_{i<n} g_{i}^{{ }^{r}}\right)=\sum_{i<n} r_{i} q_{i}
$$

( $\nu_{s}$ is well defined by the last sentence of (A) above.) Then the map

$$
w: \sum a_{f} f \mapsto \min \left\{v\left(a_{f}\right)+v_{s}(f) \mid f \in D_{s}\right\}
$$

(the coefficients $a_{f} \in F$ indexed over $f \in D_{S}$ ) is the unique valuation on $F[x]$ with $S$ an associated augmented signature.

(C) Moreover, $k_{w}=k_{S}$ and $\Gamma_{w}=\Gamma_{S} \cdot w^{-1}(\infty)=(0)$ if $S$ bas infinite degree, and $w^{-1}(\infty)=(f)$ if $S$ bas finite degree and $f$ is the generator of the unique signature $S_{0}$ with aug $S_{0}=S$.

(4.3) Corollary. Assign to each extension of $v$ to $F(x)$ the augmented signature associated with its restriction to $F[x]$. This induces a bijection from the set of equivalence classes of extensions of $v$ to $F(x)$ to the set of all signatures over $\left(k_{v}, \Gamma_{v}\right)$ with infinite degree.

Recall that a signature is an augmented signature if and only if it has infinite degree.

Proof of (4.3). Each extension of $v$ to $F(x)$ is uniquely determined by its restriction to $F[x]$ (after all, $w(a / b)=w(a)-w(b)$ for any field valuation $w$ ). A valuation on $F[x]$ extends to a valuation on $F(x)$ if and only if it has trivial infinity ideal. The corollary now follows from Theorem (4.1) and (C) of (4.2).

Suppose $w$ and $S$ correspond in the bijection of (4.3) above. Supplement (4.2) gives a picture of $w$ on $F[x]$, and hence on $F(x)$. In particular, it is easy to show that $k_{w}$ is the field of fractions of $k_{S}$ and $\Gamma_{w}$ is the group generated by $\Gamma_{S}$ (use (4.2B) to apply (4.2C)).

The remainder of this section is devoted to proving (4.1) and (4.2).

Let $w$ be an extension of $v$ to $F[x]$. There is associated with $w$ a (possibly augmented) signature of maximal length. Hence there is associated $w$ ith $w$ a unique generating sequence of maximal length (apply "(i) implies (ii)" of (3.5), together $w$ ith the definitions). Hence $w$ admits a unique (possibly augmented) signature of maximal length (reason as in the paragraph preceding (3.5)). This possibly augmented signature must in fact be augmented, for otherwise we apply (3.5) to obtain a generating sequence of greater length. This proves the first 
sentence of (4.1). Since equivalent extensions of $v$ to $F[x]$ clearly have the same generating sequences and associated (augmented) signatures, the second sentence of (4.1) follows from (4.2), which we now prove.

In the next two lemmas we suppose $S=\left\langle\theta_{i}, q_{i}\right\rangle_{i<n}$, with corresponding generating sequence $\left\langle g_{i}\right\rangle_{i<n}$, is a signature over $\left(k_{v}, \Gamma_{v}\right)$ associated $w$ ith an extension $w$ of $v$ to $F[x]$. For each $m \leq n$, let $A_{m}$ denote the multiplicative submonoid of $F[x]$ generated by $F$ and $\left\{g_{i} \mid i<m\right\}$. Note that $w$ maps $A_{m}$ onto $\Gamma_{m} \cup\{\infty\}$.

Lemma A. Suppose $m \leq n$ and $w(t) \geq 0$ for some $t \in A_{m}$. Then $t+p_{w} \in k_{m}$.

Proof. By induction it suffices to suppose the lemma holds for some $m<n$, and prove it for $m+1$. So suppose we have $w\left(t g_{m}^{s}\right)=0$ for some integer $s \geq 0$ and $t \in A_{m}$. Clearly, there is an integer $s^{\prime}$ with $s=\left(\Gamma_{m+1}: \Gamma_{m}\right) s^{\prime}$. There exist $a$ and $b$ in $A_{m}$ with

$$
w\left(a^{s^{\prime}} b\right)=0 \text { and } \theta_{m}=a g_{m}^{\left(\mathbf{r}_{m}+1: \mathbf{r}_{m}\right)}+p_{w} \text {. }
$$

Rearranging terms and using that $k_{m}$ is a field, we have

which is clearly in $k_{m+1}$. Done.

$$
t g_{m}^{s}+p_{w}=\left(a^{s^{\prime}} b+p_{w}\right)^{-1}\left(t b+p_{w}\right) \theta_{m}^{s^{\prime}}
$$

Let $D_{S}$ denote the set of products $\Pi_{i<n} g_{i}^{r_{i}}$ where only finitely many of the $r_{i}$ are nonzero and

$$
0 \leq r_{i}<\left[k_{i+1}: k_{i}\right]\left(\Gamma_{i+1}: \Gamma_{i}\right) \quad(0 \leq i<n) .
$$

This notation is consistent with our earlier use of $D_{S}$ (cf. (4.2A)).

Lemma B. Let $b_{f} \in F\left(f \in D_{S}\right)$. Then

$$
w\left(\sum b_{f} f\right)=\min _{f} w(b, f) .
$$

Proof. By induction we may suppose the lemma is true for all signatures of length $m$, where $m+1=n$. Set

$$
\gamma=\min \left\{w(b, f) \mid f \in D_{s}\right\}
$$

We may assume (throwing away all terms $b_{f} f$ with value greater that $\gamma$ and then dividing out the highest power of $g_{m}$ occurring in the remaining terms) that $\gamma \in \Gamma_{m}$ and $w\left(b_{f} f\right)=\gamma$ for all $f \in D_{S}$ with $b_{f} \neq 0$. In particular, we may assume that $q_{m}$ is commensurable with $\Gamma_{v}$, so $\left(\Gamma_{m+1}: \Gamma_{m}\right)<\infty$ and $\theta_{m} \neq 0$. Pick $a$ and $b$ in $A_{m}$ with

$$
\gamma=-w\left(b a^{\left[k_{m}+1: k_{m}\right]}\right) \text { and } \theta_{m}=a g_{m}^{\left(\mathbf{r}_{m}+1: \mathbf{r}_{m}\right)}+p_{w} .
$$

Let $S^{*}=\left\langle\theta_{i}, q_{i}\right\rangle_{i<m}$. Each $f \in D_{S}$ can be uniquely written in the form $f^{*} g_{m}^{i f}$ where $f^{*} \in D_{S^{*}}$. Now $\theta_{m}$ is a zero of no polynomial of degree less than $\left[k_{m+1}: k_{m}\right]$ over $k_{m}$. Hence 


$$
0 \neq \sum\left(\sum b a^{\left[k_{m}+1: k_{m}\right]-i} b_{f} f^{*}+k_{w}\right) \theta_{m}^{i}
$$

(the outer sum is over $i$ with $0 \leq i<\left[k_{m+1}: k_{m}\right]$, and for each such $i$, the inner sum is over $f \in D_{S}$ with $i_{f}=\left(\Gamma_{m+1}: \Gamma_{m}\right) i$. Use Lemma $A$ and the induction hypothesis to show the coefficients of $\theta_{m}^{i}$ are in $k_{m}$ and are not all zero). Hence $b a^{\left[k_{m+1}: k_{m}\right]} \sum b_{f} f+p_{w} \neq 0$ so $w\left(\sum b_{f} f\right)=-w\left(b a^{\left[k_{m+1}: k_{m}\right]}\right)=\gamma$. The lemma is proved.

Now suppose $S$ and $\left\langle g_{i}\right\rangle_{i<n}$ are as in Supplement (4.2). We claim that it suffices to show that $S$ is associated with some extension of $v$ to $F[x]$. First, (4.2A) follows immediately from (ii) of Definition (3.4) (prove the second sentence first). Notice that Lemma $B$ holds for augmented signatures $S$, using the $D_{S}$ of (4.2) (this is a corollary of Lemma B as it is stated). Then any extension of $v$ to $F[x]$ must be given by the formula of (4.2B). Finally, (4.2C) follows from (4.2B) and Lemma $A$.

First suppose $n \neq \infty$, so $n=m+1$ for some $m \geq 0$. One checks (or see Bourbaki $[2$, p. 160]) that the map

$$
w_{0}: \sum a_{i} g_{m}^{i} \mapsto \min _{i}\left(v\left(a_{i}\right)+i q_{m}\right) \quad\left(a_{i} \in F\right)
$$

defines a valuation on $F\left[g_{m}\right]$. $w_{0}$ extends to a valuation $w$ on $F[x]$ (apply [19, Proposition 7] to the extension $F[x] / F\left[g_{m}\right]$ if $q_{m}=\infty$ and to the extension $F(x) / F\left(g_{m}\right)$ if $\left.q_{m} \neq \infty\right)$. By the Fundamental Lemma (3.5) ((iii) implies (i)), $\left\langle\theta_{i}, q_{i}\right\rangle_{i<m}$ is associated $w i$ th $w$. If $q_{m}$ is not finite and commensurable with $\Gamma_{v}$, we are done. (For then, by (3.4), $S$ is associated with $w$. .) Now suppose $q_{m}$ is commensurable with $\Gamma_{\nu}$; then since $S$ is augmented, $\theta_{m}$ must be transcendental over $k_{m}$. Therefore it suffices to show that for any $a \in A_{m}\left(A_{m}\right.$ defined as above) and $t>0$ with $w\left(a g_{m}^{t}\right)=0$, we have $a g_{m}^{t}+p_{w}$ transcendental over $k_{m}$. Using Lemma $A$ we see this is the same as showing that $a g_{m}^{t}+p_{w}$ is transcendental over $k_{v}$ for $a \in F$ and $t>0$ with $w\left(a g_{m}^{t}\right)=0$. If this is not true, then for some $b_{i} \in A_{v}$ (and $v\left(b_{i}\right)=0$ for at least one $i$ ) we have

$$
\sum\left(b_{i}+p_{w}\right)\left(a g_{m}^{t}+p_{w}\right)^{i}=0,
$$

i.e. $w\left(\Sigma a^{i} b_{i} g_{m}^{t i}\right)>0$, which contradicts that $w$ is an extension of $w_{0}$.

It remains to consider the case $n=\infty$. For each $m<\infty$ let $w_{m}$ denote the unique extension associated with aug $\left\langle\theta_{i}, q_{i}\right\rangle_{i<m}$ (existence of $w_{m}$ is proved in the preceding paragraph). For any $f \in F[x]$, the sequence $\left\langle w_{i}(f)\right\rangle_{i<\infty}$ becomes constant as soon as $\operatorname{deg} g_{m}>\operatorname{deg} f$ (notice that $\operatorname{deg} S=\infty$, so $\operatorname{deg} g_{m} \rightarrow \infty$ ). The map

$$
f \mapsto \lim _{i \rightarrow \infty} w_{i}(f) \quad(f \in F[x])
$$

gives a well-defined extension of $v$ to $F[x]$, and $S$ is associated with it (use the Fundamental Lemma). 
The main theorem and its supplement are now proved. Notice that we have shown that, with notation as in (4.2), $\left\{\left\langle\theta_{i}, q_{i}\right\rangle_{i<m} \mid m \leq n\right\}$ is the set of all (possibly augmented) signatures associated with $w$. Also notice that we nowhere used in the proof of Lemmas A or B that $F$ was complete.

5. Irreducible polynomials and simple algebraic extensions. Throughout this section $F$ denotes a field complete at a discrete rank one valuation $v$. Associated signatures and generating sequences are again defined with respect to fixed systems $A$ and $B$ of representatives for $\Gamma_{v}$ and $F^{\times} /\left(1+p_{v}\right)$. As earlier, we assume $1 \in A \cap B$.

The following theorem will be applied to study the irreducible polynomials and algebraic extensions of $F$.

(5.1) Theorem. Assigning to each signature over $\left(k_{v}, \Gamma_{v}\right)$ of finite degree its generator (cf. (3.5)) gives a bijection from the set of such signatures to the set of nonconstant monic irreducible polynomials in $F[x]$.

Proof. Let $f$ be the generator of a signature $S$. Let $w$ be the unique extension of $v$ to $F[x]$ with aug $S$ associated with . $w$ (cf. (4.1)). By (3.5) and (4.2C), $f$ generates the prime ideal $w^{-1}(\infty)$. Hence $f$ is irreducible.

Conversely, suppose $f$ is a nonconstant monic irreducible polynomial. There exists an extension $w$ of $v$ to $F[x]$ with $w(f)=\infty$ (since there exists an extension of $v$ to $F[x] /(f)$ ). Then $f$ must be in the generating sequence of $w$ (apply $(4.2 \mathrm{C})$, recalling that $w^{-1}(\infty)$ can contain at most one monic irreducible polynomial). The theorem is proved.

For the remainder of this section, $f$ will denote a nonconstant monic irreducible polynomial in $F[x]$, say with corre sponding signature $S=\left\langle\theta_{i}, q_{i}\right\rangle_{i<n^{*}}$ As in the proof of (5.1), we let $w$ denote the unique extension of $v$ to $F[x]$ with $w(f)$ $=\infty$ (or equivalently, with aug $S$ associated with $w$ ). Notice that if $\theta$ is a zero of $f$ in some extension of $F$, then there is a unique extension $u$ of $v$ to $F[\theta]$ $(\cong F[x] /(f)) . u$ and $w$ are related by

$$
u(b(\theta))=w(b) \quad(b \in F[x])
$$

and have naturally isomorphic residue class fields and value groups. It is well known that $u$ (and hence $w$ ) can also be computed in terms of the norm map from $F[\theta]$ to $F$ (cf. [21, p. 53]).

(5.2) Corollary. Let $E$ be an algebraic extension of $F$. Then $v$ bas a unique extension to $E$.

Proof. For each $a \in E$, apply the above remarks to the irreducible polynomial of $a$ over $F$. 
(5.3) Corollary. Let $E$ be a finite dimensional extension of $F$. Then $E$ is complete with respect to the unique extension of $v$ to $E$.

Proof. Without loss of generality, we may suppose $E=F[x] /(f)$ (since any extension may be obtained by a sequence of simple extensions). Let $\left\langle g_{i}\right\rangle_{i<n}$ be the generating sequence of $w$ and define $D_{S}$ as in Lemma B, $\S 4$. Then $\{\bar{b}+(f) \mid$ $\left.b \in D_{S}\right\}$ is a basis for $E / F$ (indeed, an "orthobasis" in the sense of MacKenzieWhaples [14]). Any Cauchy sequence in $E,\left\langle\Sigma b_{i, b} b+(f)\right\rangle_{i<\infty}$ (for each $i$, the $b_{i, b} \in F$ are indexed over $\left.b \in D_{S}\right)$, converges to $\Sigma b_{b} b+(f)$, where, for each $b \in D_{S}, b_{b}$ is the limit of the (Cauchy!) sequence $\left\langle b_{b, i}\right\rangle_{i<\infty}$.

The above two corollaries are of course well known, as is the next, at least for algebraic extensions.

(5.4) Corollary. Let $E$ be any field extension of $F$, and let $z$ be an extension of $v$ to $E$. Then

$$
[E: F]=\left[k_{z}: k_{\nu}\right]\left(\Gamma_{z}: \Gamma_{\nu}\right)
$$

Proof. If $E / F$ is transcendental, we show that the right-hand side of (16) is infinite by the method of Corollary (4.3). If $E / F$ is algebraic, we again may assume $E=F[x] /(f)$, whence (16) follows from the equality of $\operatorname{deg} S$ and $\operatorname{deg} f$.

Notice that (16) holding for all extensions $E / F$ is necessary and sufficient for $F$ to be complete (use [12, Theorem 4]).

We now illustrate how to use (5.1) to study the irreducible polynomials over $F$. Since these results are peripheral to our main argument, we will only sketch proofs and refer the reader to [3] for a full discussion. Proofs tend to be long but straightforward computations with generating sequences.

(5.5) Lemma and Definition. There exists a least element of $\Gamma_{v}^{\mathrm{div}} \cup\{-\infty\}$, call it $\gamma_{f}$, such that, for every extension $z$ of $v$ to $F[x]$, if $z(f)>\gamma_{f}$ then $z$ and $w$ agree on all polynomials of degree less than deg $f$.

The above lemma makes sense if we identify $\Gamma_{w}$ and $\Gamma_{v}^{\text {div }}$ with their canonical images in $\Gamma_{z}^{\text {div }}$ (cf. (3.2)). Equivalently, given $\alpha \in \Gamma_{z}^{v}$ and $\beta \in \Gamma_{w} \cup \Gamma_{v}^{\text {div }}$ (so $m \beta \in \Gamma_{v}$ for a positive integer $m$ ), we set $\alpha \geq \beta$ if $m a \geq m \beta$.

The proof of (5.5) gives a computation of $\gamma_{f}$. First note that $\gamma_{f}=-\infty$ if and only if $f$ is linear (we employ the obvious conventions with $-\infty$ ). Suppose $f$ is not linear; then there exists a largest $m$ less than $n$ with $\operatorname{deg} S>\left[k_{m}: k_{\nu}\right]$ $\left(\Gamma_{m}: \Gamma_{\nu}\right)$. Let us define

$$
\gamma_{f}=\left[k_{m+1}: k_{m}\right]\left(\Gamma_{m+1}: \Gamma_{m}\right) q_{m}
$$

Then one can show that $\gamma_{f}$ satisfies the conditions of the proposition. (The 
method of proof of (3.5) shows that $z(f)>\gamma_{f}$ implies $\left\langle\theta_{i}, q_{i}\right\rangle_{i<m}$ is associated with $z$. Now use Lemma B of $\$ 4$.)

We will use $w$ and $\gamma_{f}$ to describe certain "extensions" of $f$ to an irreducible polynomial over $F$. The simplest case is treated in the next proposition, which also gives an alternate definition of $\gamma_{f}$. The lemma quantifies the well-known result that a polynomial sufficiently close to $f$ (in the sense that respective coefficients are sufficiently close in the valuation topology on $F$ ) is irreducible.

(5.6) Proposition. $\gamma_{f}$ is the least element of $\Gamma_{v}^{\text {div }} \cup\{-\infty\}$ such that any monic polynomial $g$ in $F[x]$ is irreducible if it has the same degree as $f$ and $w(f-g)>\gamma_{f}$

Note $w(f-g)=w(g) .(5.6)$ is a special case of (5.10) below.

One of the many equivalent forms of Hensel's lemma says that the canonical image in $k_{v}[x]$ of a monic irreducible polynomial whose coefficients are in $A_{v}$ is an integral power of an irreducible polynomial in $k_{v}[x]$. The following proposition generalizes this fact.

(5.7) Proposition. Suppose $f=\Sigma a_{i} x^{i}$. Then

(A) $v\left(a_{i}\right) /(n-i) \geq v\left(a_{0}\right) / n \neq \infty(i<n)$.

(B) If $e$ is the smallest positive integer with $e\left(v\left(a_{0}\right) / n\right) \in \Gamma_{v}$, say with $v(s)=(e / n) v\left(a_{0}\right)$ for $s \in F$, then

$$
y^{n / e}+\sum_{i<n / e}\left(s^{n / e-i} a_{i e}+p_{v}\right) y^{i}
$$

is an integral power of an irreducible polynomial over $k_{v}$.

Notice that (A) ensures that formula (17) makes sense. Condition (A) is well known; it says that the Newton polygon of $f$ is a straight line segment $[16, p$. 500], [21, p. 54].

To prove (5.7), write $f=g_{1}^{m}+b$ where $\operatorname{deg} f=m \operatorname{deg} g_{1}$. Then the coefficients of $b$ have sufficiently large value that the above conditions on the coefficients of $f$ can be proven by establishing the analogous conditions on the coefficients of $g_{1}^{m}$ (induct on $m$ ).

The above proposition offers formal motivation for the next definition.

(5.8) Definition. A polynomial $g \in F[x]$ is an extension of $f$ if and only if its expansion in powers of $f$ has the form

where

$$
f^{n}+\sum_{i<n} r_{i} f^{i} \quad\left(\operatorname{deg} r_{i}<\operatorname{deg} f\right)
$$

(i) $w\left(r_{i}\right) /(n-i) \geq w\left(r_{0}\right) / n>\gamma_{f}(0 \leq i<n)$;

(ii) if $e$ is the smallest positive integer with $e\left(w\left(r_{0}\right) / n\right) \in \Gamma_{w}$, say with 
$w(s)=-(e / n) w\left(r_{0}\right)$ for $s \in F[x]$, then

$$
y^{n / e}+\sum_{i<n / e}\left(s^{n / e-i} r_{i e}+p_{w}\right) y^{i}
$$

is irreducible over $k_{w}$.

Again, (i) above guarantees that (ii) makes sense. Notice that this definition could easily have been phrased in terms of the valuation $u: F[\theta] \rightarrow \Gamma_{w} \cup\{\infty\}$ of (15).

(5.9) Examples. (A) Let $g=\Sigma a_{i} x^{i}$ be a monic polynomial with coefficients $a_{i}$ in the valuation ring of $v$. If the image of $g$ in $k_{v}[x]$ is irreducible, then $g$ is an extension of $x$.

(B) Eisenstein polynomials are extensions of $x$, i.e., if $g=x^{n}+a_{n-1} x^{n-1}$ $+\cdots+a_{0}\left(a_{i} \in F\right)$ has each $a_{i} \in p_{v}$ and has $v\left(a_{0}\right)$ the smallest positive element of $\Gamma_{v}$, then $g$ is an extension of $x$. More generally, $g$ is an extension of $x$ if $v\left(a_{i}\right) /\left(x_{0}-i\right)>v\left(a_{0}\right) / n$ for $0<i<n$, and the least positive integer $s$ with $s\left(v\left(a_{0}\right) / n\right) \in \Gamma_{\nu}$ is $s=n$.

(C) Suppose $g$ satisfies all the hypotheses of Proposition (5.6). Then $g$ is an extension of $f$. In particular any polynomial of the form $x+a$ is an extension of any other such polynomial.

(D) If $\left\langle g_{i}\right\rangle_{i \leq n}$ is the generating sequence of $w$, then $g_{i+1}$ is an extension of $g_{i}$ for all $i<n$.

(5.10) Theorem. Let $g \in F[x]$. Then $g$ is a monic nonconstant irreducible polynomial if and only if there exists a finite sequence of polynomials in $F[x]$

$$
g_{0}, g_{1}, \cdots, g_{m}=g
$$

such that $\operatorname{deg} g_{0}$ equals 1 , and for each $i<m, g_{i+1}$ is an extension of $g_{i}$.

Proof (sketch). It suffices to show that $f$ admits such a sequence (18), and that any extension of $f$ is irreducible. Let $\left\langle g_{i}\right\rangle_{i \leq n}$ be the generating sequence of $w$. If $n<\infty$, we are done (cf. (5.9D)). Otherwise take the sequence (18) to be $g_{0}, g_{1}, \cdots, g_{s}, f$ where $s<n$ has $\operatorname{deg} g_{s}=\operatorname{deg} f$. Now let $g$ be an extension of $f$. Let $s$ be as large as possible with $\operatorname{deg} g_{s}<\operatorname{deg} g$. One can then extend $\left\langle\theta_{i}, q_{i}\right\rangle_{i<s}$ to a signature of which $g$ is the generator (use Lemma $G$ of $\S 8)$.

(5.10) can also be proven by using (5.5) to apply [17, Theorem 1].

Note. We may require the polynomials in (18) to have strictly increasing degree. Suppose this is done. Then the sequence (18) is still not unique. However, the value groups and residue class fields of the extensions of $v$ to $F[x] /\left(g_{i}\right)$ are invariants of $g$. For more in this direction, see [3, p. 32].

The sequence (18) can be used to compute inductively $\gamma_{g}$ and the unique extension $z$ of $v$ to $F[x]$ with $z(g)=\infty$. Suppose $g$ is an extension of $f$. Then 
$\gamma_{\boldsymbol{g}}=\gamma_{f}$ if $\operatorname{deg} g=\operatorname{deg} f$, and $\gamma_{g}=w(g)$ otherwise. Further, for any $b \in F[x]$,

$$
z(b)=\min _{i} w\left(t_{i}\right)+(i \operatorname{deg} f / \operatorname{deg} g) w(g)
$$

where

$$
\sum t_{i} f^{i} \quad\left(\operatorname{deg} t_{i}<\operatorname{deg} f\right)
$$

is the remainder upon dividing $b$ by $g$.

Let us now suppose that $b$ is a separable monic nonconstant irreducible polynomial over $F$, say associated with the signature $S^{\prime}$. It is well known that if $b$ and $f$. are sufficiently close (i.e. their respective coefficients are sufficiently close in the valuation topology of $F)$, then $F[x] /(b)$ and $F[x] /(f)$ are isomorphic over $F[20$, Chapter $F]$. We can give a quantitative statement of this result for finite dimensional tamely ramified (abbreviated, tame) extensions of $F$. Notice that $F[x] /(f)$ is a tame extension of $F$ if and only if $k_{S} / k_{\nu}$ is separable and the characteristic of $k_{v}$ does not divide $\left(\Gamma_{S}: \Gamma_{v}\right)$.

Recall $S=\left\langle\theta_{i}, q_{i}\right\rangle_{i<n}$. Let red $S$ denote the signature obtained from $S$ by deleting all terms $\left(\theta_{i}, q_{i}\right)$ with $\theta_{i} \in k_{i}$ and $q_{i} \in \Gamma_{i}$. Formally,

$$
\operatorname{red} S=\left\langle\theta_{\sigma(i)}, q_{\sigma(i)}\right\rangle_{i}<\sigma(n)
$$

where $\sigma(n)$ is the number of terms $\left(\theta_{i}, q_{i}\right)$ with $\theta_{i} \notin k_{i}$ or $q_{i} \notin \Gamma_{i}$, and, for each $i<\sigma(n),\left(\theta_{\sigma(i)}, q_{\sigma(i)}\right)$. is the $(i+1)$ th such term.

(5.11) Proposition. Suppose $F[x] /(f)$ is tamely ramified over $F$. Then $F[x] /(f)$ is isomorphic to $F[x] /(b)$ over $F$ if $\operatorname{red} S=\operatorname{red} S^{\prime}$.

Proof. Use Lemma E of $\$ 8$ to apply the proposition and first theorem of [6].

The possibility of generalizing (5.11) is suggested by the following corollaries to Krasner's lemma [20, p. 190]. The first is the well-known "p-power theorem".

(5.12) Remark. Let $p$ be a prime number. Let $a, a^{\prime} \in F$.

(A) Suppose char $F \neq p$. Then $x^{p}-a$ and $x^{p}-a^{\prime}$ give isomorphic extensions of $F$ (by adjoining a root to $F$ ) if for some $b \in F$

$$
v\left(\left(a b^{p} / a^{\prime}\right)-1\right)>p v(p) /(p-1) .
$$

(B) Suppose char $F=p$. Then $x^{p}-x-a$ and $x^{p}-x-a^{\prime}$ give isomorphic extensions of $F$ if $v\left(a-a^{\prime}\right)>0$.

These results can also be proved directly but tediously from (5.10). (One uses (5.10) to show that (19) implies $x^{p}-a^{\prime}$ is not irreducible in $F\left[a^{1 / p}\right]$, cf. [3, pp. 46-48]. (B) is similar, but easier.)

The task of giving necessary and sufficient conditions for the isomorphism of $F[x] /(f)$ and $F[x] /(b)$ in terms of $S$ and $S^{\prime}$ seems prohibitively difficult (with (5.1), this would characterize all simple algebraic extensions of $F$ in terms 
of signatures!). It is possible to give such conditions for a family of signatures whose associated generators yield all tame extensions of $F$. The resulting computation of tame extensions of $F$ shows the set of $F$-isomorphism classes of tame extensions of $F$ with specified residue class field $k$ and value group $\Gamma$ is bijective with the set of equivalence classes of elements in $k^{x} \otimes \Gamma / \Gamma_{v}$ (tensor product as abelian groups), where two elements of $k^{\times} \otimes \Gamma / \Gamma_{\nu}$ are equivalent if one is carried into the other by an automorphism of $k^{x} \otimes \Gamma / \Gamma_{v}^{v}$ induced by an automorphism of $k$ leaving $k_{v}$ fixed. (See [4].)

(5.13) Remark. In this final remark we drop the hypothesis that $F$ is complete. All of our results about irreducible polynomials (namely (5.6) through (5.10)) remain valid if we replace everywhere the word "irreducible" by "irreducible over the completion of $F$ " or even "irreducible over the Henselization of $F$ ". In particular, these results hold without modification when $F$ is Henselian.

We mention a possible application. In 1898, Stieltjes conjectured that the Legendre polynomials were irreducible over $Q$ except for obvious factors. Theorem (5.10) seems a natural tool for continuing the study of the conjecture. Indeed much of the work on the conjecture has consisted of proving that particular Legendre polynomials were irreducible over appropriate completions of $Q$. (See McCoart [13] for a summary of results.)

6. Valuations and primes on polynomial rings and rational function fields. Throughout this section $F$ will denote a field all of whose nontrivial valuations are discrete rank one, e.g. an algebraic number field, or more generally, a global field. We will assume that for each nontrivial valuation $v$ of $F$ we have fixed as usual a system of representatives for $\Gamma_{v}$ and a system of representatives for $F^{\times} /\left(1+p_{v}\right)$; associated signatures are assumed to be defined with respect to these choices.

Most of the results of this section can be generalized by rephrasing them in terms of extending a given discrete rank one valuation on an arbitrary field $K$ to $K[x]$ or $K(x)$.

For a valuation $v$ on a commutative ring $R$, let $E(v)$ denote the set of equivalence classes of extensions of $v$ to a valuation on $R[x]$. $\delta(v)$ was computed in $\S 4$ for the case $R$ a complete field. The next lemma will enable us to apply $\$ 4$ to the noncomplete case.

(6.1) Lemma. Let $\widetilde{F}$, with valuation $\tilde{v}$, be the completion of $F$ at a non. trivial valuation $v$. Restricting valuations from $\widetilde{F}[x]$ to $F[x]$ induces a well-defined map

$$
\text { Res: } \mathscr{G}(\widetilde{v}) \rightarrow \mathscr{G}(v)
$$


Res is surjective and preserves residue class rings. Also, Res maps the set of prime valuations in $\mathcal{E}(\widetilde{v})$ bijectively onto the set of prime valuations in $\mathcal{E}(v)$, and the set of valuations in $\mathcal{E}(\tilde{v})$ with trivial infinity ideal bijectively onto the set of valuations in $\mathcal{E}(v)$ with trivial infinity ideal.

One can also show that Res maps the "valuations in the sense of Manis" [19] in $\mathcal{E}(\tilde{v})$ onto the corresponding subset of $\mathcal{E}(v)$.

Before proving Lemma (6.1) at the end of this section, we make a number of easy applications. These are based on the next definition.

(6.2) Definition. Let $w$ be a valuation on $F[x]$ which restricts to a nontrival valuation, call it $v$, on $F$. By the signature associated with $w$ we mean the signature $S$ over $\left(k_{v}, \Gamma_{v}\right)$ of largest possible length $n$ such that $n \leq \infty$ and $S$ is associated (cf. (3.3)) with $w$.

While there may be many signatures associated with $w$ in the sense of Definition (3.3), the signature associated with $w$ is unique. For let $\widetilde{w} \in \mathcal{E}(\widetilde{v})$ have $\operatorname{Res}(\tilde{w})=w$ (notation as in (6.1) above). Notice that our systems of representatives for $\Gamma_{v}$ and $F^{\times} /\left(1+p_{v}\right)$ may also be regarded as systems of representatives for $\Gamma_{i}$ and $\tilde{F}^{x} /\left(1+p_{\tilde{v}}\right)$. Let $\left\langle\theta_{i}, q_{i}\right\rangle_{i<m}$ be the augmented signature associated with $\tilde{w}$, defined with respect to these systems of representatives. Let us set

$$
\begin{array}{rlrl}
t & =\infty & & \text { if } m \geq \infty, \\
& =m-1 & \text { if } q_{m-1}=\infty, m<\infty, \\
& =m & \text { if } q_{m-1} \neq \infty, m<\infty .
\end{array}
$$

Then by inspection of Definition (3.3), $\left\langle\theta_{i}, q_{i}\right\rangle_{i<t}$ is seen to be the signature associated with $w$.

(6.3) Corollary. Let $v$ be a nontrivial valuation on F. Assigning to each extension of $v$ to $F(x)$ the signature associated with its restriction to $F[x]$ induces a bijection between.the set of equivalence classes of extensions of $v$ to $F(x)$ and the set of signatures over $\left(k_{v}, \Gamma_{v}\right)$ either of length $\infty$, or of finite length and infinite degree.

Proof. Use Lemma (6.1) to apply (4.3). Notice that every signature of infinite length and finite degree extends uniquely to a signature of infinite degree.

For any finite prime $P$ of a commutative ring $R$, let us denote by $v_{P}, \Gamma_{P}$ and $k_{P}$ the unique (up to equivalence) valuation associated with $P$, its value monoid, and its residue class field, respectively (cf. Harrison $\left[10\right.$, p. 18]; $v_{P}$ is determined up to equivalence by the conditions that $\Gamma_{P}$ be a group and $\left.P=P_{v_{P}}\right)$.

(6.4) Corollary (the primes of $F[x])$. Assign to each finite prime $P$ of $F[x]$ 
the signature associated with $v_{P}(c f .(6.2))$. This gives a bijection between the finite primes of $F[x]$ and the set of pairs $(S, T)$ where $T$ is a finite prime of $F$ and $S$ is a signature over $\left(k_{T}, \Gamma_{T}\right)$ with $\Gamma_{S}$ a group and $k_{S}$ a field.

(The above conditions on $S$ are equivalent to every element of $\Gamma_{S}$ being commensurable with $\Gamma_{T}$, and every element of $k_{S}$ being algebraic over $k_{T}$.)

Proof. The valuations $w$ on $F[x]$ associated with finite primes are those with $\Gamma_{w}$ a group and $k_{w}$ a locally finite field. Use (6.1) to apply (4.1) and (4.2) to the family of such valuations.

We remark that if $(S, T)$ and $P$ correspond as in (6.4), then $P \cap F=T$, and we have natural isomorphisms $k_{S} \cong k_{P}$ and $\Gamma_{S} \cong \Gamma_{P}$. The theorem of $\$ 2$ follows from the above Corollary (also use (4.2) to show that the $p$-signature associated with a prime valuation $w$ in $\$ 2$ is the signature associated with $w$ in the sense of (6.2)).

An argument similar to that establishing (6.4) gives

(6.5) Corollary (the primes of $F(x)$ ). Assign to eacb finite prime $P$ of $F(x)$ the signature associated with the restriction of $v_{P}$ to $F[x]$. This gives a bijection from the finite primes of $F(x)$ onto the set of pairs $(S, T)$ where $T$ is a finite prime of $F$ and $S$ is a signature over $\left(k_{T}, \Gamma_{T}\right)$ such that $k_{S}$ is a field and $S$ has either length $\infty$, or finite length and infinite degree.

Combining (6.4) and (6.5) we obtain a result which is well known for finite fields $F([8],[10])$.

(6.6) Corollary. Every finite prime of $F[x]$ is contained in one, and only one, prime of $F(x)$.

One can show that if $P$ is a finite prime of $F(x)$, then $P \cap F[x]$ is a prime of $F[x]$ if and only if the rank and rational rank [2, pp. 114 and 163] of $\Gamma_{P}$ are the same.

(6.7) Remark. Let us suppose in this remark that $F$ is a global field and that $R$ is a ring whose field of fractions is $F$. We use (6.4) to study the finite primes of $R[x]$.

First, the primes of $R[x]$ which are ideals (i.e. "ideal primes") all have the form $p \cdot R[x]+f \cdot R[x]$ where $p$ is an ideal prime of $R$ and $f \in R[x]$ is irreducible and nonconstant modulo $p$. Hence the ideal primes of $R[x]$ correspond bijectively to the signatures over $(R / h,\{0\})$ of finite degree, where $\{0\}$ denotes the trivial ordered group and $p$ ranges over the ideal primes of $R$.

Now let $P$ be a finite prime of $R[x]$ which is not an ideal. Then there is a unique prime $T$ of $F[x]$ containing $P$, and $v_{T}$ is the unique extension of $v_{P}$ to a valuation on $F[x]$ (use here that $\Gamma_{T}$ has rational rank one). Hence we have a 
bijection between the finite nonideal primes of $R[x]$ and the signatures of primes $T$ of $F[x]$ such that $v_{T}$ restricts to a prime valuation of $R[x]$.

Things now become simpler if we assume that $F$ is an algebraic number field (otherwise $F[x]$ can admit finite primes with trivial intersection with $R[x]$ !). Then every finite prime of $F[x]$ intersects down to a prime of $R[x]$ (use the fact that every prime of $F[x]$ contains a nonzero prime number). Hence the nonideal finite primes of $R[x]$ correspond bijectively with signatures $S$ over $\left(k_{P}, \Gamma_{P}\right)$ where $P$ ranges over the finite primes of $F, \Gamma_{S}$ is a group, $k_{S}$ is a field, and either $R$ is not contained in the valuation ring of $v_{P}$, or else $q_{0}<0$ (here, $\left(\theta_{0}, q_{0}\right)$ denotes the "first term" of $\left.S\right)$.

A concrete example. The primes of $Z[x]$ which are ideals correspond to signatures over $\left(Z_{p},\{0\}\right)$ of finite degree, $p$ ranging over all prime numbers. The finite primes of $Z[x]$ which are not ideals correspond to signatures $\left\langle\theta_{i}, q_{i}\right\rangle_{i<n}$ over $\left(Z_{p}, Z\right)$ where $p$ is any prime number, $n \leq \infty$, each $q_{i}$ is commensurable with $Z$, each $\theta_{i}$ is algebraic over $Z_{p}$, and $q_{0}<0$ if $0<n$.

(6.8) Remark. Let $P$ be a finite prime of $F[x]$, say with associated signature $S$. Let $\widetilde{F}$ with valuation $\widetilde{v}$ be the completion of $F$ at the restriction of $v_{P}$ to $F$; by (6.1) there is a unique prime $\widetilde{P}$ of $\widetilde{F}[x]$ with $\widetilde{P} \supseteq P \cup A_{\tilde{v}} \cdot P$ and $\widetilde{P}$ have the same residue class field and value group, and we can get a pretty good idea of the internal structure of the primed ring $(F[x], P)$ from the picture that Supplement (4.2) provides of $(\tilde{F}[x], \widetilde{P})$ (notice that aug $S$ is the augmented signature associated with $\left.v_{\tilde{P}_{\tilde{P}}}\right)$. Incidentally, one can give a formula for $v_{P}$ without referring explicitly to $\widetilde{F}$ by a limiting process similar to that in formula (14) at the end of $\$ 4$; see $[3$, p. 20] or $[15$, p. 372] for details.

Associated signatures also give an external picture of sets of finite primes. For example, if we give the set of finite primes of $F(x)$ the topology in which a typical basic open set is the collection of finite primes containing a given finite subset of $F$, then the bijection of (6.5) carries this topology into a transparent topology on the set of signatures associated with primes of $F(x)[5, \S 10]$. In [5], an approximation theorem is proven for compact subsets of the space of finite primes of a field which generalizes (among other things) the classical approximation theorem for inequivalent valuations.

Let notation be as in (6.1). In our next and final remark we describe how much is lost when a valuation in $\varepsilon(\tilde{v})$ is restricted to $F[x]$. In particular, when do inequivalent valuations in $\mathcal{E}(\tilde{v})$ restrict to equivalent valuations on $F[x]$, and what happens to value monoids under the mapping Res (recall that Res preserved residue class fields)? Notice that answering the first question gives a computation of $\mathscr{E}(v)$ (since Res is surjective and $\mathscr{E}(\tilde{v})$ is computed in $\S 4$ ).

(6.9) Remark. Notation is as in (6.1). Let $w \in \mathcal{E}(\tilde{v})$. 
First suppose the associated signature of $w$ (i.e. signature of maximal length $\leq \infty$ which is associated with $w$, cf. (6.2)) is not a signature of length $\infty$ and finite degree. Then $w$ is the unique extension of $\operatorname{Res}(w)$ in $\mathcal{E}(\tilde{v}), w$ and $\operatorname{Res}(w)$ have the same value group, and the infinity ideal of $w$ is generated as an ideal of $\widetilde{F}[x]$ by the infinity ideal of $\operatorname{Res}(w)$.

Next suppose that the associated signature $S$ of $w$ has finite degree and length $\infty$. Let $g_{\infty} \in \tilde{F}[x]$ be its generator. We have two cases.

Case I. $g_{\infty}$ divides (in $\tilde{F}[x]$ ) no nonzero polynomial whose coefficients are in $F$ (equivalently, one of the coefficients of $g_{\infty}$ is transcendental over $F$ ). Then $\operatorname{Res}(w)$ has exactly two extensions to an element of $\mathcal{E}(\tilde{v})$ (corresponding to the two augmented signatures extending $S$ ), the infinity ideal of $\operatorname{Res}(w)$ is trivial, $k_{S}=k_{w}$, and $\Gamma_{S}=\Gamma_{w}$.

Case II. $g_{\infty}$ divides some monic irreducible $f \in F[x]$. Then $w$ is the unique extension of $\operatorname{Res}(w)$ to an element of $\mathscr{E}(\tilde{v}), k_{\operatorname{Res}(w)}=k_{w}$, and the following six conditions are equivalent: (1) $w(f)=\infty$. (2) $\Gamma_{w}$ is a group. (3) $\Gamma_{w}=\Gamma_{s}$. (4) Aug $S$ is the augmented signature of $w$. (5) The infinity ideal of Res $(w)$ is nontrivial. (6) The infinity ideal of $\operatorname{Res}(w)$ is $(f)$. When none of the above conditions hold, then $\Gamma_{\operatorname{Res}(w)}$ is generated by $\Gamma_{S}$ and $w(f)$, and $w(f)$ is finite and larger than every element of $\Gamma_{S}$.

We prove (6.1) and (6.9) together. For related results about extensions of valuations, see [3, p. 68].

Let $w \in \mathcal{E}(v)$. $w$ induces a valuation $u$ on the field of fractions $E$ of $F[x] / w^{-1}(\infty)$. There exists an algebraically closed maximal field $\left(E^{\prime}, u^{\prime}\right)$ containing $(E, u)$ (that is, $E^{\prime} \supseteq E$ and $u^{\prime}$ extends $u$ ). By a simple transfinite construction there exists a maximal extension $\left(E^{\prime \prime}, u^{\prime \prime}\right)$ of $(\tilde{F}, \tilde{v})$ with the same value group and residue class field as $u^{\prime}$. By Kaplansky [12, Theorem 7], there exists a value preserving isomorphism leaving $F$ elementwise fixed,

$$
\Theta:\left(E^{\prime}, u^{\prime}\right) \rightarrow\left(E^{\prime \prime}, u^{\prime \prime}\right) \text {. }
$$

Now define $\tilde{w} \in \tilde{E}(\tilde{v})$ by

$$
\widetilde{w}: \sum a_{i} x^{i} \mapsto u^{\prime \prime}\left(\sum a_{i} \Theta\left(x^{i}+w^{-i}(\infty)\right)\right) \quad\left(a_{i} \in F\right) .
$$

Clearly $\operatorname{Res}(\tilde{w})=w$.

This proves that the map Res is surjective. One now can check (6.9) directly using $\$ 4$. The rest of (6.1) follows from (6.9).

7. Linearly compact fields. In this section we sketch a generalization of the results of $\$ \S 3,4$ and 5 on fields complete at a discrete rank one valuation to fields which are linearly compact at a valuation of arbitrary rank. A valued field is linearly compact (alias, maximal, ultracomplete) when its additive group 
is complete at every (not necessarily Hausdorff) topology admitting a family of fractional ideals as a neighborhood system of zero. (See [2], [9], [20], [21].)

Our notion of a sequence must be generalized. For any ordinal number $\lambda$, the expression $\left\langle a_{\nu}\right\rangle_{\nu<\lambda}$ denotes the function defined on the set of ordinal numbers less than $\lambda$ whose value at $\nu$ is $a_{\nu}$; we call it a sequence of length $\lambda$. The reader may find it convenient to regard each ordinal number as the set of ordinal numbers less than it, but we use no special properties of this "representation" of the ordinal numbers. For any ordinal number $\lambda$, we denote by $\lambda+1$ its successor. 0 denotes the first ordinal number.

Now let $F$ with valuation $v$ be a linearly compact field. Let $A$ be a system of representatives in $F^{\times}$for $\Gamma_{\nu}$. Instead of picking a system of representatives for $F^{\times} /\left(1+p_{v}\right)$, we pick a "display" $\Phi$ for $v$ in the sense of the following proposition.

(7.1) Proposition and Definition. Let $W(v)$ denote the set of all well ordered subsets of $F^{\times} /\left(1+p_{v}\right)$, where we write $a\left(1+p_{v}\right)<b\left(1+p_{v}\right)$ if and only if $v(a)<v(b)$. Then there exists a bijection $\Phi: F \rightarrow W(v)$ such that for any $a \in F^{\times}$ and $b \in F$ we bave

(i) $a\left(1+p_{v}\right)$ is the least element of $\Phi(a)$,

(ii) if every element of $\Phi(b)$ is less than every element of $\Phi(a)$, then $\Phi(a+b)=\Phi(a) \cup \Phi(b)$.

We call such a map $\Phi$ a display of $v$.

(7.2) Remark. (A) Suppose $F=Q_{p}$ (the $p$-adic numbers). Then

$$
\sum a_{i} p^{i} \mapsto\left\{a_{i} p^{i}\left(1+p_{v}\right) \mid i \text { an integer with } a_{i} \neq 0\right\} \text { : }
$$

is a display of $v$ (here $0 \leq a_{i}<p$ for all $i$ ). The situation for power series fields is even simpler.

(B) Suppose $v$ is discrete rank one. Then the set of displays of $v$ is bijective with the set of systems of coset representatives in $F^{\times}$for $F^{\times} /\left(1+p_{v}\right)$ (correspond to each display the set of elements of $F^{\times}$whose images under the display are singletons).

(C) $W(v)$ can naturally be given the structure of a valued field [3, p. 22]; it is then isomorphic to $\Delta F$ (cf. [6]) which is (not naturally) isomorphic to the generalized power series field $S\left(k_{v}, \Gamma_{v}, f\right)[21$, p. 23]. Here $f$ is any factor set whose cohomology class in $\operatorname{Ext}\left(\Gamma_{v}, k_{v}^{\times}\right)$corresponds to the exact sequence

$$
1 \rightarrow k_{v}^{\times} \rightarrow F^{\times} /\left(1+p_{v}\right) \rightarrow \Gamma_{v} \rightarrow 0
$$

(D) When $F$ is not necessarily linearly compact, there exists an injection $\Phi: F \rightarrow W(v)$ satisfying (i) and (ii) of (7.1) and such that $\Phi(F)$ is closed under 
the taking of initial and terminal segments. Such a "generalized display" is surjective if and only if $F$ is linearly compact (this fact is essentially due to Carruth [7]).

The construction of $\Phi$ is a long but basically routine exercise in transfinite induction, much like Krull's proof of the existence of maximal immediate extensions [21, pp. 37-38]. We refer the reader to $[3, \$ 5]$ for details and a fuller discussion of the remarks above.

Our choice of $\Phi$ serves two purposes. It gives us a system of representatives for $F^{\times} /\left(1+p_{\nu}\right)$ (namely the elements of $F$ which $\Phi$ maps to singletons) and it allows us to assign a specific pseudolimit to each psuedoconvergent sequence $[21$, p. 39] in $F$ (essentially, the unique pseudolimit whose image in $W(v)$ has minimal order type).

We now define "signatures over $\left(k_{v}, \mathrm{I}_{v}\right)$ " by strict analogy with Definition (3.1) (but allowing our sequences to be indexed over ordinal numbers). That is, a presignature over $\left(k_{\nu}, \Gamma_{\nu}\right)$ is a sequence of ordered pairs $S=\left\langle\theta_{\nu}, q_{\nu}\right\rangle_{\nu<\lambda}$ where the $\theta_{\nu}$ lie in some field containing $k_{\nu}$ and the $q_{\nu}$ lie in some ordered group containing $\Gamma_{\nu}$. Given such a presignature $S$, we let $k_{\mu}=k_{\nu}\left[\left\{\theta_{\nu} \mid \nu<\mu\right\}\right]$ and let $\Gamma_{\mu}$ be the semigroup generated by $\Gamma_{\nu}$ and $\left\{q_{\nu} \mid \nu<\mu\right\}$, for any $\mu \leq \lambda$. Set $k_{S}=k_{\lambda}$ and $\Gamma_{S}=\Gamma_{\lambda}$. Presignatures $S$ and $S^{\prime}$ over $\left(k_{v}, \Gamma_{v}\right)$ are equivalent if there are isomorphisms of $k_{S}$ and $\Gamma_{S}$ onto $k_{S}$, and $\Gamma_{S}$ ' leaving $k_{v}$ and $\Gamma_{v}$ fixed, which map each $\theta_{\nu}$ to $\theta_{\nu}^{\prime}$ and $q_{\nu}$ to $q_{\nu}^{\prime}$.

(7.3) Definition. A signature over $\left(k_{v}, \Gamma_{v}\right)$ is an equivalence class of presignatures $S$ with (notation as above):

(i) if $\nu<\lambda$, then $\left[k_{\nu}: k_{\nu}\right]\left(\Gamma_{\nu}: \Gamma_{\nu}\right)<\infty$, and the sequence $\left\langle q_{\nu} /\left[k_{\nu}: k_{\nu}\right]\left(\Gamma_{\nu}: \Gamma_{\nu}\right)\right\rangle_{\nu<\lambda}$ is strictly increasing;

(ii) if $\nu<\lambda$, then $\theta_{\nu}$ is nonzero if and only if $q_{\nu}$ is commensurable with $\Gamma_{\nu}$.

The sequence of (i) above of course lies in $\Gamma_{S}^{\text {div }}$ (cf. (3.2)).

If one considers $\infty$ as the first infinite ordinal number and $\infty+1$ as its successor, then the signatures of Definition (3.1) are signatures in the above sense, and if $\Gamma_{v}$ is discrete rank one, then every signature in the above sense is a signature in the sense of (3.1).

(7.4) Notation. Let $S=\left\langle\theta_{\nu}, q_{\nu}\right\rangle_{\nu<\lambda}$ be a signature over $\left(k_{\nu}, \Gamma{ }_{\nu}\right)$. We define $\operatorname{deg} S$, aug $S, k_{\text {aug } S}$, and $\Gamma_{\text {aug } S}$ by strict analogy with Definition (3.3) (i.e., just as (7.3) was obtained from (3.1) by allowing $i$ and $n$ to be ordinal numbers). Given a sequence of polynomials $g \cdot\left\langle g_{\nu}\right\rangle_{\nu<\lambda}$, we define (by analogy with the notation of Lemma $B, S 4) D(S, g)$ to be the set of all products $I I_{\nu<\lambda} g_{\nu}^{r}$ where all but a finite number of the $r_{\nu}$ are zero and

$$
0 \leq r_{\nu}<\left[k_{\nu+1}: k_{\nu}\right]\left(\Gamma_{\nu+1}: \Gamma_{\nu}\right) \quad(\nu<\lambda) .
$$


If $\lambda=0$, we also set $D(S, g)=\{1\}$. When there is no danger of ambiguity, we write

$$
D(\mu)=D\left(\left\langle\theta_{\nu}, q_{\nu}\right\rangle_{\nu<\mu},\left\langle g_{\nu}\right\rangle_{\nu<\mu}\right) \quad(\mu \leq \lambda)
$$

Finally, if $S$ is an augmented signature, we still define $D(\mu)$ as in (21) for $\mu$ less than the length of $S$.

(7.5) Definition. Let $w \in \mathcal{E}(v)$ (cf. \$6). A (possibly augmented) signature $S=\left\langle\theta_{\nu}, q_{\nu}\right\rangle_{\nu<\lambda}$ over $\left(k_{v}, \Gamma_{\nu}\right)$ is associated with $w$ if and only if there exists a sequence $\left\langle g_{\nu}\right\rangle_{\nu<\lambda}$ in $F[x]$ (called a generating sequence for $w$ and $S$ ) such that

(i) if $0<\lambda$, then $g_{0}=x$;

(ii) if $\mu+1<\lambda$, then we can write

$$
g_{\mu+1}=g_{\mu}^{\left[k_{\mu+1}: k_{\mu}\right]\left(\boldsymbol{\Gamma}_{\mu+1}: \boldsymbol{\Gamma}_{\mu}\right)}+\sum_{f \in D(\mu+1)} b_{\mu, f} f
$$

where each nonzero term on the right-hand side of (22) has the same value, and for each nonzero coefficient $b_{\mu, f}, \Phi\left(b_{\mu, f}\right)$ is a singleton;

(iii) if $\mu<\lambda$, then we can write

$$
g_{\mu}=g_{\mu^{*}}+\sum_{f \in D(\mu)} d_{\mu, f} f
$$

where $\mu^{*}$ is least with $\operatorname{deg} g_{\mu}=\operatorname{deg} g_{\mu^{*}}$ and for each $f \in D(\mu)$

$$
\Phi\left(d_{\mu, f}\right)=\bigcup_{\mu^{*} \leq \nu<\mu} \Phi\left(b_{\nu, f}\right) ;
$$

(iv) if $\mu<\lambda$, then $q_{\mu}=w\left(g_{\mu}\right)$;

(v) if $\mu<\lambda$ and $q_{\mu}$ is finite and commensurable with $\Gamma_{v}$, then

$$
\theta_{\mu}=\left(a \prod_{\nu \leq \mu} g_{\nu}^{r_{\nu}}\right) g_{\mu}+P_{w}
$$

where $a \in A$ and $0 \leq r_{\nu}<\left(\Gamma_{\nu+1}: \Gamma_{\nu}\right)$ for all $\nu \leq \mu$.

As in $\$ 3$, a sequence $g$ of monic polynomials in $F[x]$ is called a generating sequence for $w$ when there exists a (possibly augmented) signature $S$ such that $g$ is a generating sequence for $w$ and $S$.

Let $\mu<\lambda . D(\mu)$ is an $F$-module basis for the set of polynomials in $F[x]$ of degree less than $\operatorname{deg} g_{\mu}$. This is because the degree of $g_{\nu}$ is clearly $\left[k_{\nu}: k_{\nu}\right]\left(\Gamma_{\nu}: \Gamma_{\nu}\right)$ for each $\nu<\lambda$. Hence the coefficients $b_{\mu, f}$ and $d_{\mu, f}$ of (22) and (23) above are uniquely determined, so that (24) is unambiguous.

It is not hard to show that this definition generalizes (3.4); for this we assume, of course, that $\Phi$ has been chosen so that $\Phi(b)$ is a singleton for each $b \in B$ (cf. (7.2B)). The real point is that the $\infty$-term of a generating sequence in the sense of (3.4), if there is one, is obtained by the limiting process of (iii) above.

We now make a blanket assertion. With the above definitions, all the results 
of $\$ \S 3,4$ and 5 about a field complete at a discrete rank one valuation hold for any linearly compact field. Specifically, we have generalizations of the following.

1. The Fundamental Lemma (3.5) (of course one allows the indices $i$ and $n$ in (3.5) to be ordinal numbers).

2. The Main Theorem, its Supplement, and Corollary (in $\$ 4$ ).

3. Theorem (5.1) on irreducible polynomials.

4. Corollaries (5.2), (5.3), and (5.4) on algebraic extensions. Again, the condition that (16) hold for arbitrary field extensions $E / F$ is necessary and sufficient for $F$ to be linearly compact.

5. Propositions (5.5), (5.6) and (5.7), Definition (5.8), the Examples (5.9), and Theorem (5.10). Of course we do not replace $i$ and $n$ here by ordinal numbers (except in (5.9D)).

6. Remarks (5.11) and (5.12) hold, as well as the bijection mentioned after (5.12) from the $F$-isomorphism classes of tame extensions of $F$ with residue class field $k$ and value group $\Gamma$ onto the equivalence classes in the tensor product $\Gamma / \Gamma_{v} \otimes k^{\times}$.

We will prove the generalized Fundamental Lemma in $\S 8$. The other results require only a routine generalization of the arguments given above in the discrete rank one case. A minor exception is Corollary (5.3), which uses the criterion for linear compactness mentioned in (7.2D). Details can be found in [3].

We were able in $\$ 6$ to apply our results on complete fields to noncomplete fields. No such generalization seems possible in general. Similarly no analogue to Remark (5.13) seems available. One can show that if $\tilde{F}$ with valuation $\tilde{v}$ is a maximal immediate extension of a not necessarily linearly compact field $F$ (with valuation $v$ ), then the obvious map Res: $\mathcal{E}(\tilde{v}) \rightarrow \mathcal{E}(v)$ is surjective. One can get a fair insight into the possible extensions of $v$ to $F[x]$ and $F(x)$ by using this surjection to apply our computation of $\mathcal{E}(\tilde{v})$ (e.g. one can read off an inductive proof of Proposition (2.46) of [1]).

8. Proof of the Fundamental Lemma. Let $w$ be a valuation on a commutative ring $R$. Following Mac Lane [15], we will call elements $a$ and $b$ of $R$ equiv. alent in $w$ when either $w(a-b)>w(a)$ or $w(a)=w(b)=\infty$. For $a \in R$ we denote by $[a]_{w}$ the set of elements of $R$ equivalent in $w$ to $a$. Multiplication preserves equivalence and we have a nonoid with cancellation

$$
C_{w}=\left\{[a]_{w} \mid a \in R, w(a) \neq \infty\right\} .
$$

Note that if $w(a)=0$, then $[a]_{w}=a+p_{w}$, so $k_{w}^{\times} \subseteq C_{w}\left(k_{w}^{\times}\right.$denotes the multiplicative monoid of nonzero elements of $\left.k_{w}\right)$. Let $w$ operate on $C_{w}$ by $w\left([a]_{w}\right)=$ $w(a)$; we then have an exact sequence of monoids

$$
1 \rightarrow k_{w}^{\times} \rightarrow C_{w} \rightarrow \Gamma_{u} \rightarrow 0 .
$$


Clearly, $C_{w}$ is a group if and only if $\Gamma_{w}$ is a group and $k_{w}$ is a field. If $R$ is a field, then $C_{w}=R^{x} /\left(1+p_{w}\right)$.

Equivalence plays an essential role in the proof of the Fundamental Lemma. Its role in our earlier exposition has been important, if only implicit, e.g. the key condition (III) in $\S 2$ says $g_{m+1}$ can be written in a certain way as a difference of equivalent polynomials.

We now prove the Fundamental Lemma, generalized to linearly compact fields. So let $F, \nu, \Phi$, and $A$ be as in $\$ 7$. Let $\left\langle\theta_{\nu}, q_{\nu}\right\rangle_{\nu<0}$ be a signature of finite degree. By induction on $\rho$, we may suppose we have a sequence $\left\langle g_{\nu}\right\rangle_{\nu<\rho}$ of monic nonconstant polynomials in $F[x]$ such that for any $\lambda<\rho$ and $w \in E(v)$, the following are equivalent:

(I) $\left\langle\theta_{\nu}, q_{\nu}\right\rangle_{\nu<\lambda}$ is associated with $w$;

(II) $\left\langle g_{\nu}\right\rangle_{\nu \leq \lambda}$ is the unique generating sequence for $w$ of length $\lambda+1$;

(III) $w\left(g_{\lambda}\right)>\left[k_{\nu}: k_{\nu}\right]\left(\Gamma_{\nu}: \Gamma_{\nu}\right) q_{\nu}$ for all $\nu<\lambda$.

It suffices to construct a monic nonconstant $g_{\rho} \in F[x]$ such that for any $w \in \mathcal{E}(\nu)$, the above three conditions are equivalent with $\lambda=\rho$.

Let $\lambda \leq \rho$. In the next five lemmas we let $w \in \mathcal{E}(v)$ denote any valuation with which $S=\left\langle\theta_{\nu}, q_{\nu}\right\rangle_{\nu<\lambda}$ is associated. The existence of such $w$ is guaranteed when $\lambda<\rho$ by our induction hypothesis (consider any $w \in \mathcal{E}(v)$ with $\left.w\left(g_{\lambda}\right)=\infty\right)$. We let $F_{\boldsymbol{\lambda}}$ denote the set of polynomials in $F[x]$ of degree less than $\operatorname{deg} g_{\lambda}$, and set

$$
C_{w}(\lambda)=\left\{[a]_{w} \mid 0 \neq a \in F_{\lambda}\right\}
$$

The structure of $C_{w}(\lambda)$ is computed in Lemma E below.

By our induction hypothesis $D(\lambda)$ is a basis for $F_{\lambda}$ considered as an $F$-module (cf. (7.4)). For each $f=\mathrm{II}_{\nu<\lambda} g_{\nu}^{r}$ in $D(\lambda)$, we set $v_{\lambda}(f)=\Sigma r_{\nu} q_{\nu}$ (just as in (4.2B); we of course assume the $r_{\nu}$ satisfy (20)). The next lemma says that $w$ is completely determined on $F_{\lambda}$ by $S$.

Lemma C. For any $a, \in F(f \in D(\lambda))$,

$$
w\left(\sum a_{f} f\right)=\min \left(v\left(a_{f}\right)+v_{\lambda}(f)\right)
$$

Proof. By our induction hypothesis, $\left\langle g_{\nu}\right\rangle_{\nu<\lambda}$ is a generating sequence for $w$. Now proceed as in the proof of Lemma B of $\$ 4$. Done.

Let $B(\lambda)$ denote the set of polynomials $\Sigma a_{f} f$ in $F_{\lambda}$ such that each nonzero coefficient $a_{f}$ has $\Phi\left(a_{f}\right)$ a singleton, and each nonzero term $a_{f} f$ has the same value. By the preceding lemma, $B(\lambda)$ depends only on $S$ (i.e. is independent of the choice of $w$ ) and is a system of representatives in $F_{\lambda}$ for $C_{w}(\lambda)$. 
For each $\mu<\rho$, let $e_{\mu}=\left(\Gamma_{\mu+1}: \Gamma_{\mu}\right)$ and $f_{\mu}=\left[k_{\mu_{+1}}: k_{\mu}\right]$. Let $A(\mu)$ denote the set of polynomials

$$
a \prod_{\nu<\mu} g_{\nu}^{r_{\nu}}
$$

where $a \in A$ and $0 \leq r_{\nu}<e_{\nu}$ for $\nu<\mu$. There exists a unique $a_{\mu} \in A(\mu)$ which when written as in (25) has $v(a)+\Sigma r_{\nu} q_{\nu}+e_{\mu} q_{\mu}=0$. Notice that $\theta_{\mu}=a_{\mu} g_{\mu}^{e_{\mu}}+$ $\mathrm{P}_{w}$ if $\mu<\lambda$, and $w$ maps $A(\lambda)$ bijectively to $\Gamma_{\lambda}$.

Lemma D. $C_{w}(\lambda)$ is a subgroup of $C_{w}$. Also, $w\left(C_{w}(\lambda)\right)=\Gamma_{\lambda}$ and $k_{w}^{\times} \cap C_{w}(\lambda)=k_{\lambda}^{\times}$.

Proof. Induct on $\lambda$. Lemma $C$ implies $w\left(C_{w}(\lambda)\right)=\Gamma_{\lambda}$. Lemma $A(\S 4)$ generalizes to our situation (same proof), so clearly $k_{w}^{x} \cap C_{w}(\lambda) \subseteq k_{\lambda}^{x} \cdot F_{\lambda}$ is closed under addition, so the reverse implication follows if any $\theta=\left(b+p_{w}\right) \theta_{\mu}^{i}$ $\left(\mu<\lambda, i<f_{\mu}, b \in F_{\mu}\right.$ and $\left.w(b)=0\right)$ is in $C_{w}(\lambda) . C_{w}(\mu)$ is a group by induction, so $[f]_{w}=$ $\left[b a_{\mu}^{i}\right]_{w}$ for some $f \in F_{\mu}$. Since $f g_{\mu}^{i e} \mu \in F_{\lambda}, \theta=f g_{\mu}^{i e} \mu+p_{w} \in C_{w}(\lambda)$. That $C_{w}(\lambda)$ is a group now follows from the exactness of the sequence of monoids

$$
1 \rightarrow k_{\lambda}^{\times} \rightarrow C_{w}(\lambda) \rightarrow \Gamma_{\lambda} \rightarrow 0
$$

The lemma is proved.

For each $a \in \Gamma_{v}$, let $a_{\alpha}$ be the unique element of $A$ with $v\left(a_{a}\right)=\alpha$. More generally, for $\alpha \in \Gamma_{\lambda}$, let $a_{a}$ be the unique element of $A(\lambda)$ of the form (25) with $v(a)+\Sigma r_{\nu} q_{\nu}=a$.

We now define a map

$$
\varphi_{\lambda}: \Gamma_{\lambda} \times \Gamma_{\lambda} \rightarrow k_{\lambda}^{\times}
$$

by induction on $\lambda$. If $\lambda=0$, we define $\boldsymbol{\varphi}_{\lambda}$ by

$$
\boldsymbol{\varphi}_{0}(\alpha, \beta)=a_{\alpha} a_{\beta} a_{a+\beta}^{-1}+p_{w} \quad\left(\alpha, \beta \in \Gamma_{v}\right) .
$$

Suppose $\lambda=\mu+1$. Let $\alpha, \beta \in \Gamma_{\mu}$ and let $r$ and $s$ be nonnegative integers less than $e_{\mu}$. We then define $\varphi_{\lambda}\left(\alpha+r q_{\mu}, \beta+s q_{\mu}\right)$ to be $\varphi_{\mu}(\alpha, \beta)$ if $r+s<e_{\mu}$ and to be $\varphi_{\mu}(\alpha, \beta) \theta_{\mu} / \varphi_{\mu}\left(-e_{\mu} q_{\mu}, \alpha+\beta+e_{\mu} q_{\mu}\right)$ otherwise. Finally, if $\lambda$ is a limit ordinal, let $\varphi_{\lambda}$ be the union of all the maps $\varphi_{\mu}, \mu<\lambda$.

$\varphi_{\lambda}$ induces a (not necessarily associative) multiplication on the direct product $C_{\lambda}=k_{\lambda}^{\times} \times \Gamma_{\lambda}$, namely

$$
(\theta, q) \cdot\left(\theta^{\prime}, q^{\prime}\right)=\left(\varphi_{\lambda}\left(q, q^{\prime}\right) \theta \theta^{\prime}, q+q^{\prime}\right) .
$$

The sequence

$$
1 \rightarrow k_{\lambda}^{\times} \rightarrow C_{\lambda} \rightarrow \Gamma_{\lambda} \rightarrow 0
$$


preserves multiplication and is exact (i.e. "image = kernel" everywhere).

Lemma E. $C_{\lambda}$ is a group, and the map

$$
\alpha \mapsto\left(\alpha \cdot\left[a_{w(\alpha)}\right]_{w}^{-1}, w(\alpha)\right) \quad\left(\alpha \in C_{w}(\lambda)\right)
$$

is an isomorphism from $C_{w}(\lambda)$ to $C_{\lambda}$.

Proof. The above map is a bijection (use Lemma D), so it suffices to show it preserves multiplication. The set of $\left[a_{\alpha}\right]_{w}\left(a \in \Gamma_{\lambda}\right)$ is a system of representatives in $C_{w}(\lambda)$ for $\Gamma_{\lambda}$. A straightforward (inductive) computation shows that the corresponding factor set for the group extension (26) is exactly $\boldsymbol{\varphi}_{\lambda}$, proving the lemma. (For factor sets and group extensions, see Mac Lane [18, p. 111].)

Lemma F. Suppose $w^{\prime} \in \mathcal{E}(v)$ is associated with $S$. We identify $k_{\lambda}$ with its canonical images in $k_{w}$ and $k_{w}$ ' (thus we identify $a_{\mu} g_{\mu}^{e_{\mu}}+p_{w}$ with $a_{\mu} g_{\mu}^{e_{\mu}}+$ $p_{w}{ }^{\prime}$ for all $\left.\mu<\lambda\right)$. Then the correspondence $[a]_{w} \leftrightarrow[a]_{w}^{\prime}\left(a \in F_{\lambda}, a \neq 0\right)$ is a group isomorphism between $C_{w}(\lambda)$ and $C_{w},(\lambda)$ which is the identity on $k_{\lambda}^{x}$.

Proof. We also identify $\Gamma_{\lambda}$ with its canonical image in $\Gamma_{w}$ and $\Gamma_{w}{ }^{\prime}$. By Lemma $C$, we then have $w(b)=w^{\prime}(b)$ for all $b \in F_{\lambda}$. Let us inductively suppose the lemma true for all $\mu<\lambda$. It suffices to show that

$$
[b]_{w}\left[a_{w(b)}\right]_{w}^{-1}=[b]_{w},\left[a_{w(b)}\right]_{w^{\prime}}^{-1}
$$

for any $0 \neq b \in F_{\lambda}$, since we may then apply Lemma $\mathrm{E}$ to $w$ and $w^{\prime}$. It suffices to consider $b$ of the form $c g_{\mu}^{n e \mu+m}$ where $c \in F_{\mu}, m<e_{\mu}$ and $n<f_{\mu}$. Then $a_{w(b)}=a^{\prime} g_{\mu}^{m}, a^{\prime} \in A(\mu)$. The left-hand side of $(28)$ is $[c]_{w}\left[a^{\prime}\right]_{w}^{-1}\left[a_{\mu}\right]_{w}^{-n} \theta_{\mu}^{n}$, which by hypothesis is independent of $w$, and hence equal to the right-hand side of (28). Done.

Lemma G. Suppose $\lambda+1=\rho$ and that $\left\langle\theta_{\nu}, q_{\nu}\right\rangle_{\nu<\rho}$ is associated with w. Then a polynomial of the form

$$
g_{\lambda}^{e_{\lambda} f_{\lambda}}+\sum_{i<f_{\lambda}} b_{i} g_{\lambda}^{i e_{\lambda}}
$$

(where for each $i<f_{\lambda}$, if $b_{i} \neq 0$ then $b_{i} \in B(\lambda)$ and $\left.w\left(b_{i}\right)=\left(f_{\lambda}-i\right) e_{\lambda} q_{\lambda}\right)$ is the $\rho$ th term of a generating sequence for $w$ if and only if

$$
y^{f_{\lambda}}+\sum_{i<f_{\lambda}}\left(a_{\lambda}^{f_{\lambda}-i} b_{i}+p_{w}\right) y^{i}
$$

is the monic irreducible polynomial over $k_{\lambda}$ with $\theta_{\lambda}$ as a zero. If this is indeed the case, then $b_{0} \neq 0$.

Proof. The last sentence follows from the fact that the constant term of the irreducible polynomial of $\theta_{\boldsymbol{\lambda}}$ over $k_{\lambda}$ is nonzero.

Let $g_{\rho}$ be as in (29). If it is in the generating sequence for $w$, then 


$$
0=a_{\lambda}^{f_{\lambda}} g_{\rho}+p_{w}=\theta_{\lambda}^{f \lambda}+\sum\left(a_{\lambda}^{f_{\lambda}-i} b_{i}+p_{w}\right) \theta_{\lambda}^{i}
$$

so (30) is indeed the irreducible polynomial of $\theta_{\lambda}$ over $k_{\lambda}$. Conversely, if (30) is this irreducible polynomial, then $w\left(g_{\rho}\right)>-w\left(a_{\lambda} f_{\lambda}\right)=e_{\lambda} f_{\lambda} q_{\lambda}$, so the lemma follows by inspection of Definition (7.5).

We now construct $g_{\rho}$. For each $\mu+1<\rho$, we have unique $b_{\mu, f}(f \in D(\mu+1))$ with $g_{\mu+1}=g_{\mu}^{e f \mu}+\Sigma b_{\mu, f} f$ (cf. (22)). If $\rho$ is a limit ordinal, let $\lambda<\rho$ be smallest with $\Gamma_{\boldsymbol{\lambda}}=\Gamma_{\rho}$ and $k_{\lambda}=k_{\rho}$. Define $d_{\rho, f} \in F$ for each $f \in D(\lambda)$ by

$$
\Phi\left(d_{\rho, f}\right)=\bigcup_{\lambda \leq \nu<\rho} \Phi\left(b_{\nu, f}\right) .
$$

We then set $g_{\rho}=g_{\lambda}+\Sigma d_{\rho, f} f$. If $\rho$ is not a limit ordinal, say $\rho=\lambda+1$, we can pick $b_{i} \in B(\lambda) \cup\{0\}$ such that, for any $w \in E(v)$ with which $\left\langle\theta_{\nu}, q_{\nu}\right\rangle_{\nu<\lambda}$ is associated,

$$
y^{f_{\lambda}}+\sum_{i<f_{\lambda}}\left(a_{\lambda}^{f_{\lambda}{ }^{-i} b_{i}}+p_{w}\right) y^{i}
$$

is the irreducible polynomial of $\theta_{\lambda}$ over $k_{\lambda}$, and the value of $b_{i}$ is $\left(f_{\lambda}-i\right) e_{\lambda} q_{\lambda}$ whenever $b_{i} \neq 0$. The existence and uniqueness of the $b_{i}$ follow from Lemmas $\mathrm{D}$ and $\mathrm{F}$. We set

$$
g_{\rho}=g_{\lambda}^{e_{\lambda} f_{\lambda}}+\sum_{i<f_{\lambda}} b_{i} g_{\lambda}^{i e_{\lambda}}
$$

which can be uniquely written in the form

$$
g_{\lambda}^{e_{\lambda} f_{\lambda}}+\sum_{f \in D(\rho)} b_{\lambda, f} f
$$

Now let $w \in \mathcal{E}(v)$. We prove our three conditions are equivalent with $\lambda=\rho$.

(I) implies (II). By induction, $\left\langle g_{\nu}\right\rangle_{\nu<\rho}$ is the unique generating sequence of $w$ of length $\rho$. First suppose $w$ is a limit ordinal. Then one checks (II) using Definition (7.5) (the key fact is that $u\left(g_{\rho}\right)>q_{\nu}$ for all sufficiently large $\nu<\rho)$. Next, suppose $\rho=\lambda+1$. (II) then follows by Lemma $G$ and our construction of $g_{\rho}$.

(II) implies (III). (III) follows by induction if $\rho$ is a limit ordinal. If $\rho=\lambda+1$, then $\left\langle\theta_{\nu}, q_{\nu}\right\rangle_{\nu<\lambda}$ is associated with $w$. Let $f$ be the remainder obtained when dividing $g_{\rho}$ by $g_{\lambda}$. By the last sentence of Lemma G (applied to whatever signature $w$ has), we have $w\left(g_{\rho}\right)>w(f)=e_{\lambda} f_{\lambda} q_{\lambda}$, which proves (III).

(III) implies (I). First suppose $u\left(g_{\nu}\right)=q_{\nu}$ for all $\nu<\rho$. We are then done by induction if $\rho$ is a limit ordinal, so suppose $\rho=\lambda+1$. Then $\left\langle\theta_{\nu}, q_{\nu}\right\rangle_{\nu<\lambda}$ is associated with $w$, so it suffices to show that $a_{\lambda} g_{\lambda}^{\boldsymbol{e}_{\lambda}}+p_{w}$ satisfies the same irreducible polynomial over $k_{\lambda}$ as $\theta_{\lambda}$ does (cf. (3.1)). But since $w\left(g_{\rho}\right)>$ $e_{\lambda} f_{\lambda} q_{\lambda}$, this follows by our construction of $g_{\rho}$. 
Now suppose that for some least $t<\rho, w\left(g_{t}\right) \neq q_{t}$. We are done if we obtain a contradiction. Let $0=\lambda_{0}<\lambda_{1}<\cdots<\lambda_{n}<\lambda_{n+1}=\rho$ be a finite sequence of ordinals containing $\left\{\nu<\rho \mid e_{\nu} f_{\nu} \neq 1\right\} \cup\{t\}$. For the remainder of this section we will identify the nonnegative integer $i$ with the ordinal number $\lambda_{i}$; we regard $i+1$ as denoting $\lambda_{i+1}$ and not $\lambda_{i}+1$. For each $0 \leq i \leq n$ we have unique $c_{i, f}$ $(f \in D(i+1))$ with $g_{i+1}=g_{i}^{e_{i} f_{i}}+\Sigma c_{i, f} f$. Evidently, for each $f \in D(i+1)$,

$$
\Phi\left(c_{i, f}\right)=\bigcup \Phi\left(b_{\nu, f}\right) \quad \text { (union over } i \leq \nu<i+1 \text { ) }
$$

and, by the last sentence of Lemma G, for some $b=\Pi g_{\nu}^{s \nu} \in D(i)$,

$$
v\left(c_{i, b}\right)+\sum_{\nu<i} s_{\nu} q_{\nu}=e_{i} f_{i} q_{i}
$$

Claim 1. $w\left(g_{t}\right)>q_{t}$, so $\left\langle\theta_{\nu}, q_{\nu}\right\rangle_{\nu<t}$ is associated with $w$.

Proof. Suppose not. Then $w\left(g_{t}\right)<q_{t}$, so $w\left(g_{i}\right)<q_{i}$ for $t \leq i \leq n$ (use the induction hypothesis on $\left.g_{i}\right)$. We claim $w\left(g_{j+1}\right)=e_{j} f_{j} w\left(g_{j}\right)$ for $t \leq j \leq n$. Suppose inductively that this is true for all $i<j$, where $t \leq j \leq n$. For each $f=\Pi_{\nu_{\leq j}} g_{\nu}^{r \nu}$ $\epsilon D(j+1)$,

$$
\begin{aligned}
w\left(c_{j, f} f\right) & \geq e_{j} f_{j} q_{j}+w(f)-\sum_{0 \leq i \leq j} r_{i} q_{i} \geq e_{j} f_{j} q_{j}+\sum_{0 \leq i \leq j}\left(e_{i} f_{i}-1\right)\left(w\left(g_{i}\right)-q_{i}\right) \\
& \geq e_{j} f_{j} w\left(g_{j}\right)+q_{t}-w\left(g_{t}\right)+\sum_{t \leq i \leq j} q_{i+1}-e_{i} f_{i} q_{i}>e_{j} f_{j} w\left(g_{j}\right)=w\left(g_{j}{ }^{f_{j}}\right)
\end{aligned}
$$

which implies $w\left(g_{j+1}\right)=e_{j} f_{j} w\left(g_{j}\right)$. Now set $j=n$ to get $w\left(g_{n+1}\right)=e_{n} f_{n} w\left(g_{n}\right)$. Hence $w\left(g_{n}\right)>q_{n}$. Since $w\left(g_{t}\right)<q_{t}$, we cannot have $n>t$; hence $n=t$ and the claim is proved.

Claim 2. $n>t$ and $w\left(g_{t+1}\right)=e_{t} f_{t} q_{t}$.

The first assertion follows from the second by (III). Using (31) and Claim 1 we have $w\left(\Sigma_{f \in D(t)} c_{t, f} f\right)=e_{t} f_{t} q_{t}$. Writing

$$
g_{t+1}=\left(\sum_{f \in D(t)} c_{t, f} f\right)+\left(g_{t}^{e_{t} f_{t}}+\sum_{f \notin D(t)} c_{t, f} f\right)
$$

we see $w\left(g_{t+1}\right)=e_{t} f_{t} q_{t}$. (Use that the second term of (32) has value greater than $e_{t} f_{t} q_{t}$ by Claim 1.)

Claim 3. $w\left(g_{j+1}\right)=e_{j} f_{j} w\left(g_{j}\right)$ for all $t<j \leq n$.

Proof. Suppose the claim holds for all $i<j$. For each $f \in B(j+1)$ we have $w\left(c_{j, f} f\right)>e_{j} f_{j} w\left(g_{j}\right)$ (a straightforward computation along the lines of that in Claim 1). The claim follows immediately.

If in Claim 3 we take $j=n$, then $w\left(g_{n+1}\right)=e_{n} f_{n} w\left(g_{n}\right)$. By Claim 2, $n>t$, so $w\left(g_{n}\right)<q_{n}$. Hence $w\left(g_{n+1}\right)<e_{n} f_{n} q_{n}$, contradicting (III).

The Fundamental Lemma is proved.

The Lemmas C, D, E and F can be proven in much greater generality [3, p. 78]. 
It is easy to apply them to compute $C_{w}$ for any extension $w$ of $v$ to $F[x]$ or $F(x)$.

9. Polynomials in several variables. Let $F$ be a global field. In this brief section we sketch a computation of the finite primes of $F[x, y]$ which have discrete rank one value group (abbreviated, discrete primes). This allows us to illustrate how the methods of $\$ 6$ can be applied to commutative rings, and also gives an application of our remarks on equivalence in $\$ 8$. As in $\$ 6$, we assume we have chosen systems of representatives for the value group $\Gamma_{\nu}$ and "equivalence class group" $C_{v}$ of each nontrivial valuation $v$ of $F$. For more on valuations on rings of polynomials in several variables see Mac Lane $[17, \$ 6]$ and Inoue [11].

First note that a discrete prime of $F[x, y]$ restricts to a discrete prime of $F[x]$. The discrete primes of $F[x]$ are computed in $\$ 6$; hence it suffices to calculate the extensions of such a prime $P$ to a discrete prime of $F[x, y]$. Now for any valuation $v$ on a commutative ring $R, \mathcal{E}(v)$ is naturally bijective with $\mathscr{E}\left(v^{*}\right)$, where $v^{*}$ is the valuation induced by $v$ on the field of fractions of $R / v^{-1}(\infty)$. Thus it suffices to compute the extensions of $u$ to a prime discrete rank one valuation on $E[y]$, where $u$ is the valuation induced by $v_{P}$ on the field of fractions $E$ of $F[x] / v_{P}^{-1}(\infty)$. By our remarks in $\S 8$, the chosen systems of representatives for the value group and equivalence class group of the restriction of $v_{P}$ to $F$ extend canonically to systems of representatives for $\Gamma_{P}$ and $C_{v_{P}}$ (recall " $A(\lambda)$ and $\left.B(\lambda)^{\prime \prime}\right)$, thence giving systems of representatives in $E^{\times}$for $\Gamma_{u}$ and $C_{u}$. We can then compute the discrete rank one prime valuations in $\mathcal{E}(u)$ by defining associated signatures (cf. (6.2)) with respect to these systems of representatives.

Putting all this together, we obtain a bijection between the discrete primes of $F[x, y]$ and the set of triples $(P, S, T)$ where $P$ is a finite prime of $F, S$ is a signature over $\left(k_{P}, \Gamma_{P}\right)$ with $\left(\Gamma_{S}: \Gamma_{P}\right)<\infty$ and $k_{S}$ a field, and $T$ is a signature over $\left(k_{S}, \Gamma_{S}\right)$ with $\left(\Gamma_{T}: \Gamma_{S}\right)<\infty$ and $k_{T}$ a field. This bijection is natural modulo the choice of systems of representatives in $F^{\times}$for the value groups and equivalence class groups of valuations on $F$. The bijection can be iterated in the obvious way to any number of variables.

10. Mac Lane's inductive and limit values. We now give the connections between the concepts and results above and those in Mac Lane ([15], [16], [17]). We delayed until now the detailed acknowledgement of $M$ ac Lane's work because of the rather technical nature of these connections.

Assume $v$ is a discrete rank one valuation on a field $F$, and that we have chosen systems of representatives $A$ and $B$ in $F^{\times}$for $\Gamma_{v}$ and $C_{v}$ with $1 \in A \cap B$. We shall regard $\Gamma_{v}$ as contained in the ordered additive group of real numbers $R$. We also regard all rank one valuations as taking values in $\mathbf{R} \cup\{\infty\}$.

We review the construction in [15] of extensions of $v$ to $F[x]$, using slightly 
different notation. For any $\mu \in \mathbf{R} \cup\{\infty\}$, we let $[v ;(x, \mu)]$ denote the valuation on $F[x]$,

$$
\sum r_{i} x^{i} \mapsto \min _{i}\left(v\left(r_{i}\right)+i \mu\right) \quad\left(r_{i} \in F\right)
$$

Next (the induction step), let $w$ be any rank one valuation on $F[x]$ extending $v$. Suppose $\phi \in F[x]$ and $\mu \in \mathbf{R} \cup\{\infty\}$ satisfy

(i) $\phi$ has minimal degree in its equivalence class $[\phi]_{w}$;

(ii) $[\phi]_{w}$ is irreducible in the semigroup $C_{w}$;

(iii) $\mu>w(\phi)$.

We understand (ii) to exclude the possibility that $[\phi]_{w}$ is a unit in $C_{w}$. We then let $[w ;(\phi, \mu)]$ denote the valuation on $F[x]$ which maps a polynomial (expanded in powers of $\phi) \sum r_{i} \phi^{i}\left(\operatorname{deg} r_{i}<\operatorname{deg} \phi\right)$ to $\min _{i}\left(w\left(r_{i}\right)+i \mu\right) .[w ;(\phi, \mu)]$ is called an augmentation of $w$ (cf. [15, Theorem (4.2)]). $\phi$ is called a key polynomial for $w$.

We now define, for appropriate sequences $\left\langle\mu_{i}\right\rangle_{i<n}$ in $\mathbf{R} \cup\{\infty\}$ and $\left\langle\phi_{i}\right\rangle_{i<n}$ in $F[x]$, where $1 \leq n \leq \infty$, a valuation denoted by

$$
\left[v ;\left\langle\phi_{i}, \mu_{i}\right\rangle_{i<n}\right] .
$$

For finite $n,(33)$ is defined inductively by the formula

$$
\left[v ;\left\langle\phi_{i}, \mu_{i}\right\rangle_{i<m+1}\right]=\left[\left[v ;\left\langle\phi_{i}, \mu_{i}\right\rangle_{i<m}\right] ;\left(\phi_{m}, \mu_{m}\right)\right]
$$

where we let $\left[v ;\left\langle\phi_{i}, \mu_{i}\right\rangle_{i<0}\right]=v$. Such valuations Mac Lane calls inductive values. If $n=\infty$, we define (33) to be the limit of the $\left[v ;\left\langle\phi_{i}, \mu_{i}\right\rangle_{i<m}\right]$ as $m \rightarrow$ $\infty$. That is, any $f \in F[x]$ is assigned the value $\lim _{m \rightarrow \infty}\left[v ;\left\langle\phi_{i}, \mu_{i}\right\rangle_{i<m}\right](f)$. These valuations Mac Lane calls limit values. The conditions that must be placed on the $\phi_{i}$ and $\mu_{i}$ are simply those ensuring that (34) always be defined (i.e. see (i), (ii), (iii) above).

One says that the sequence $\left\langle\phi_{i}, \mu_{i}\right\rangle_{i<n}$ gives a bomogeneous representation of the valuation $\left[v ;\left\langle\phi_{i}, \mu_{i}\right\rangle_{i<n}\right]$ if the $\phi_{i}$ are distinct polynomials of nondecreasing degree and, for $0<m<n, \phi_{m}$ is monic and $\left[v ;\left\langle\phi_{i}, \mu_{i}\right\rangle_{i<m}\right]$-homogeneous in $\left\langle p_{i}\right\rangle_{i<m}$ (cf. \$3). Then [15, Theorems (16.2) and (16.3)] every extension of $v$ to a rank one valuation on $F[x]$ bas a unique homogeneous representation.

(Strictly speaking, Mac Lane only considers "finite" values in [15], i.e. valuations with trivial infinity ideals, but the above result follows immediately from his methods. Some caution is indicated when allowing nonfinite values, e.g. his [15, Corollary (16.4)] is false in this case.)

The following two propositions allow one to translate the language of generators and signatures into that of inductive and limit values. Let $w$ be an extension of $v$ to a rank one valuation on $F[x]$, say with homogeneous representation 
$\left[\nu ;\left\langle\phi_{i}, \mu_{i}\right\rangle_{i<m}\right]$. Let $\left\langle\theta_{i}, q_{i}\right\rangle_{i<n}$ be the signature associated with $w$ (cf. (6.2)) and let $\left\langle g_{i}\right\rangle_{i<n}$ be the corresponding generating sequence.

Proposition A. The sequences $\left\langle\phi_{i}, \mu_{i}\right\rangle_{i<m}$ and $\left\langle g_{i}, q_{i}\right\rangle_{i<n}$ are identical.

Proposition B. $w$ admits an augmentation if and only if $C_{w}$ is not a group, and bence if and only if, for some $s<n,\left[k_{s+1}: k_{s}\right]\left(\Gamma_{s+1}: \Gamma_{s}\right)=\infty$ (necessarily then, $s+1=n)$. The key polynomials over $w$ are exactly the polynomials $\phi$ which are either equivalent in $w$ to $g_{s}$, or equivalent in $w$ to the generator of $a$ signature identical with $\left\langle\theta_{i}, q_{i}\right\rangle_{i<\imath}$ except for $\theta_{s}$. Of course, equivalent key polynomials give the same augmentations.

(The second case, i.e. $\phi$ not equivalent in $w$ to $g_{s}$, arises only if $\theta_{s}$ is transcendental over $k_{s}$ and is the only case in which $\phi$ is a key polynomial for an inductive value in the sense of [ 15, Theorem (9.4)].)

Let notation be as above. For each $m<n, \phi_{m}$ is the unique monic $f \in F[x]$ of least degree which is $w$-homogeneous in $\left\langle\phi_{i}\right\rangle_{i<m}$ and has

$$
w(f)>\left[v ;\left\langle\phi_{i}, \mu_{i}\right\rangle_{i<m}\right](f) .
$$

Using (4.2) (and (6.1) of course) and assuming inductively that, for some $m<n$, $\left\langle\phi_{i}, \mu_{i}\right\rangle_{i<m}=\left\langle g_{i}, q_{i}\right\rangle_{i<m}$, one checks that $f=g_{m}$ satisfies (35), so $g_{m}=\phi_{m}$. Hence $q_{m}=w\left(g_{m}\right)=w\left(\phi_{m}\right)=\mu_{m}$. This proves Proposition A. The first sentence of B follows from (4.2) and (6.1). The second sentence is a corollary of Proposition A (consider the homogeneous representation of $w$ and its augmentation). With a little more work one can prove the existence and uniqueness of homogeneous representations, and hence the above two propositions, using only the results of this paper.

With the above two propositions, all the theorems of [15] with the exception of the interesting but peripheral [15, Theorem (6.5)] can be proved as corollaries to the results of this paper. (A second exception must be also made for fundamental elementary results like [15, Theorem (2.1)] which we have treated as common property.) With somewhat more effort, our Theorems (4.1) and (4.2) can be proved using the methods of [15]; the argument centers around the careful inductive application of [15, Theorems (12.1) and (13.1)]. The rank two valuations do not fit nicely into the framework of [15] and must be handled on an ad hoc basis. For example, [15, Theorem (16.1)] is false if one allows nonfinite and rank two valuations. (For $F$ complete, [15, Theorem (16.1)] is true but [15, Theorem (16.2)] fails.) The trouble comes from valuations with generating sequences of length $\infty+1$, where the polynomial $g_{\infty}$ can take on either a finite value or value $\infty$. Homogeneous representations do not distinguish between these possibilities. This was the reason of course for considering sequences of length 
$\infty+1$. Part of the fundamental lemma can also be proven with the methods of [15], but the crucial assertion (that (iii) of (3.5) implies (i)) seems to require the elementary but complicated counting argument of $\$ 8$.

In discussing irreducibility, Mac Lane starts from the observation that the key polynomials over an extension of $v$ to $F[x]$ are irreducible. This fairly obvious fact includes a large number of "polygonal irreducibility criteria" (cf. $[17, \S 5])$-the strength of the fact lies in our knowledge of the extensions themselves. When $F$ is locally compact, $\mathrm{Mac}$ Lane obtains a method for testing in a finite number of steps the irreducibility of a polynomial in $F[x]$. Of course this is only implicit in his papers since he never (except in [15, Theorem (7.1)]) mentions locally compact or even complete fields. The irreducibility criterion we give can of course be proven from Mac Lane's if one assumes the Fundamental Lemma (or, more precisely, (5.5) together with the computation of $\gamma_{f}$ ).

\section{REFERENCES}

1. S. Abhyankar, Ramification theoretic methods in algebraic geometry, Ann. of Math. Studies, no. 43, Princeton Univ. Press, Princeton, N. J., 1959. MR 21 \#4158.

2. N. Bourbaki, Éléments de mathématique. Fasc. XXX. Algèbre commutative.

Chap. 6: Valuations, Actualités Sci. Indust., no. 1308, Hermann, Paris, 1964. MR 33 \#2660.

3. Ron Brown, Irreducibility over complete rings, Thesis, University of Oregon, Eugene, Ore., 1968.

4. - Tume extensions of linearly compact fields (in preparation).

5. - An approximation theorem for extended absolute values, Canad. J. Math. 24 (1972), 167-184.

6. Ron Brown and D. K. Harrison, Tamely ramified extensions of linearly compact fields, J. Algebra 15 (1970), 371-375.

7. P. W. Carruth, Generalized power series fields, Trans. Amer. Math. Soc. 63 (1948), 548-559. MR 9, 561.

8. J. W. S. Cassels and A. Frölich, Algebraic number theory, Proc. Instructional Conf. Organized by the London Math. Soc. (A NATO Advanced Study Institute), supported by the Internat. Math. Union, Academic Press, London; Thompson, Washington, D. C., 1967. MR $35 \# 6500$.

9. I. Fleischer, Completeness in valued spaces and algebras, Quart. J. Math. Oxford Ser. (2) 15 (1964), 345-348. MR 31 \#2241.

10. D. K. Harrison, Finite and infinite primes for rings and fields, Mem. Amer. Math. Soc. No. 68 (1966).

11. H. Inoue, On valuations of polynomial rings of many variables. I, J. Fac. Sci. Hokkaido Univ. Ser. I 21 (1970), 46-74. MR 41 \#410.

12. I. Kaplansky, Maximal fields with valuations, Duke Math. J. 9 (1942), 303-321. MR 3, 264.

13. R. F. MacCoart, Irreducibility of certain classes of Legendre polynomials, Duke Math. J. 28 (1961), 239-246.

14. R. E. MacKenzie and G. Whaples, Artin-Schreier equations in characteristic zero, Amer. J. Math. 78 (1956), 473-485. MR 19, 834.

15. S. Mac Lane, $A$ construction for absolute values on polynomial rings, Trans. Amer. Math. Soc. 40 (1936), 363-395.

16. - A construction for prime ideals as absolute values of an algebraic field, Duke Math. J. 2 (1936), 492-510. 
17. S. Mac Lane, The Schönemann-Eisenstein irreducibility criteria in terms of prime ideals, Trans. Amer. Math. Soc. 43 (1938), 226-239.

18. - Homology, Die Grundlehren der math. Wissenschaften, Band 114, Academic Press, New York; Springer-Verlag, Berlin, 1963. MR 28 \#122.

19. M. E. Manis, Valuations on a commutative ring, Proc. Amer. Math. Soc. 20 (1969), 193-198. MR 38 \#2134.

20. P. Ribenboim, Théorie des valuations, 2ième éd., Séminaire de Mathématiques Supérieures, no. 9 (Été, 1964), Les Presses de l'Université de Montréal, Montréal, Que., 1968. MR 40 \#2670.

21. O. F. G. Schilling, The theory of valuations, Math. Surveys, no. 4, Amer. Math. Soc., Providence, R. I., 1950. MR 13, 315.

DEPARTMENT OF MATHEMATICS, UNIVERSITY OF OREGON, EUGENE, OREGON 97403

DEPARTMENT OF MATHEMATICS, SIMON FRASER UNIVERSITY, BURNABY, B. C., CANADA 96822

Current address: Department of Mathematics, University of Hawaii, Honolulu, Hawaii 